\title{
Two Designs of Space-Time Block Codes Achieving Full Diversity with Partial
}

\section{Interference Cancellation Group Decoding}

\author{
Wei Zhang, Member, IEEE, Tianyi Xu, Student Member, IEEE, and \\ Xiang-Gen Xia, Fellow, IEEE
}

\begin{abstract}
A partial interference cancellation (PIC) group decoding based space-time block code (STBC) design criterion was recently proposed by Guo and Xia, where the decoding complexity and the code rate trade-off is dealt when the full diversity is achieved. In this paper, two designs of STBC are proposed for any number of transmit antennas that can obtain full diversity when a PIC group decoding (with a particular grouping scheme) is applied at receiver. With the PIC group decoding and an appropriate grouping scheme for the decoding, the proposed STBC are shown to obtain the same diversity gain as the ML decoding, but have a low decoding complexity. The first proposed STBC is designed with multiple diagonal layers and it can obtain the full diversity for two-layer design with the PIC group decoding and the rate is up to 2 symbols per channel use. But with PIC-SIC group decoding, the first proposed STBC can obtain full diversity for any number of layers and the rate can be full. The second proposed STBC can obtain full diversity and a rate up to 9/4 with the PIC group decoding. Some code design examples are given and simulation results show that the newly proposed STBC can well address the rate-performance-complexity tradeoff of the MIMO systems.
\end{abstract}

\section{Index Terms}

W. Zhang is with School of Electrical Engineering and Telecommunications, University of New South Wales, Sydney, Australia (e-mail: wzhang@ee.unsw.edu.au). His work was supported in part by the Australian Research Council Discovery Project DP1094194.

T. Xu and X.-G. Xia are with Department of Electrical and Computer Engineering, University of Delaware, DE 19716, USA (e-mail: $\{$ txu, xxia $\} @$ ee.udel.edu). Their work was supported in part by the Air Force Office of Scientific Research (AFOSR) under Grant No. FA9550-08-1-0219.

This work was presented in part at the IEEE Global Telecommunications Conference, Hawaii, USA, December 2009. 
Diversity techniques, space-time block codes, linear receiver, partial interference cancellation.

\section{INTRODUCTION}

Space-time (ST) coding is a bandwidth-efficient transmission technique that can improve the reliability of data transmission in MIMO wireless systems [1], [2]. Orthogonal space-time block coding (OSTBC) is one of the most attractive ST coding approaches because the special structure of orthogonality guarantees a full diversity and a simple (linear) maximum-likelihood (ML) decoding. The first OSTBC design was proposed by Alamouti in [1] for two transmit antennas and was then extended by Tarokh et. al. in [2] for any number of transmit antennas. A class of OSTBC from complex design with the code rate of $1 / 2$ was also given by Tarokh et. al. in [2]. Later, systematic constructions of complex OSTBC of rates $(k+1) /(2 k)$ for $M=2 k-1$ or $M=2 k$ transmit antennas for any positive integer $k$ were proposed in [3] $-[5]$. However, the OSTBC has a low code rate not more than $3 / 4$ for more than two transmit antennas [6].

To enhance the transmission rate of the STBC, various STBC design approaches were proposed such as quasi-OSTBC [7]-[9], [11] $-[13],[15]-[18]$ and algebraic number theory based STBC [19]-[27]. The quasi-OSTBC increases the code rate by relaxing the orthogonality condition on the code matrix, which was originally proposed in [7], [8], and [9], independently. Due to the group orthogonality, the ML decoding is performed pair-wise or group-wise with an increased complexity compared to the single-symbol decoding. In [14]-[16], quasi-OSTBC was studied in the sense of minimum decoding complexity, i.e., a real pair-wise symbols decoding. In [16][18], the pair-wise decoding was generalized to a general group-wise decoding. The decoding for these codes is the ML decoding and their rates are basically limited by that of OSTBC. The algebraic number theory based STBC are designed mainly based on the ML decoding that may have high complexity and even though some near-ML decoder, such as sphere decoder [28] can be used, the expected decoding complexity is still dominated by polynomial terms of a number of symbols which are jointly detected [29].

To reduce the large decoding complexity of the high rate STBC aforementioned, several fastdecodable STBC were recently proposed [30] [31]. The STBC proposed in [30] achieves a high rate and a reduced decoding complexity at the cost of loss of full diversity. The fast-decodable STBC in [31] can obtain full rate, full diversity and the reduced ML decoding complexity, but the code design is limited to $2 \times 2$ and $4 \times 2$ MIMO transmissions only. Another new 
perspective of reducing the decoding complexity was recently considered in [33] and [34] to resort to conventional linear receivers such as zero-forcing $(\mathrm{ZF})$ receiver or minimum mean square error (MMSE) receiver instead of the ML receiver to collect the full diversity. The outage and diversity of linear receivers in flat-fading MIMO channels were studied in [32], but no explicit code design was given to achieve the full diversity when the linear receivers are used. Based on the new STBC design criterion for MIMO systems with linear receivers, Toeplitz STBC [33] and overlapped-Alamouti codes [34] were proposed and shown to achieve the full diversity with the linear receivers. Recently, some other new designs of STBC with linear receivers were proposed [35]-[37]. However, the code rate of STBC achieving full diversity with linear receivers is upper bounded by one. Later, Guo and Xia proposed a partial interference cancellation (PIC) group decoding scheme [38] which can be viewed as an intermediate decoding approach between the ML receiver and the ZF receiver by trading a simple single-symbol decoding complexity for a high code rate larger than one symbol per channel use. Moreover, in [38] an STBC design criterion was given to achieve full diversity when the PIC group decoding is applied at the receiver. The proposed PIC group decoding in [38] was also connected with the successive interference cancellation (SIC) strategy to aid the decoding process, referred to as PIC-SIC group decoding. A few code design examples were presented in [38], but a general design of STBC achieving full diversity with the PIC group decoding remains an open problem.

In this paper, we propose two designs of STBC which can achieve full diversity with the PIC group decoding for any number of transmit antennas. The first proposed STBC have a structure of multiple diagonal layers and for each diagonal layer there are exactly $M$ coded symbols embedded, being equal to the number of transmit antennas, which are obtained from a cyclotomic lattice design. Indeed, each diagonal layer of the coded symbols can be viewed as the conventional rate-one diagonal STBC [39], [40]. The code rate of the proposed STBC can be from one to $M$ symbols per channel use by adjusting the codeword length, i.e., embedding different number of layers in the code matrix. With the PIC group decoding the code rate of the first proposed full-diversity STBC can be only up to 2 symbols per channel use, i.e., for two layers. For more than two layers embedded in the codeword, the code rate is increased at the cost of losing full diversity with the PIC group decoding. However, with the PIC-SIC group decoding, the proposed STBC with arbitrary number of layers can obtain full diversity and the code rate can be up to $M$. 
The second proposed STBC is designed with three layers of information symbols embedded in the codeword and the PIC group decoding can be performed in three separate groups accordingly. Without loss of decoding complexity compared to the first proposed STBC, the second proposed STBC can achieve full diversity and a code rate larger than 2 . Note that the code rate for the first proposed full-diversity STBC with PIC group decoding can not be above 2. In the PIC group decoding of the proposed STBC, every $M$ neighboring columns of the equivalent channel matrix are clustered into one group.

This paper is organized as follows. A system model of ST transmission over MIMO channels with the PIC group decoding is introduced in Section [II. In Section [II, a design of high rate STBC with the PIC group decoding is proposed, which contains multiple diagonal layers of coded symbols. For a particular code design with two diagonal layers, the full diversity with the PIC group decoding is proved. For the code with PIC-SIC group decoding, the full diversity is shown for any number of diagonal layers. Several full-diversity code design examples are given in Section IV. In Section V, another design of high rate STBC with the PIC group decoding is proposed, which can achieve full diversity with three layers. Simulation results are presented in Section VI, Finally, in Section VII, we draw our conclusions.

Notations: Column vectors (matrices) are denoted by boldface lower (upper) case letters. Superscripts ${ }^{t}$ and ${ }^{H}$ stand for transpose and conjugate transpose, respectively. $\mathbb{C}$ denotes the field of complex numbers. $\mathbf{I}_{n}$ denotes the $n \times n$ identity matrix, and $\mathbf{0}_{m \times n}$ denotes the $m \times n$ matrix whose elements are all $0 . \operatorname{vec}(\mathbf{X})$ is the vectorization of matrix $\mathbf{X}$ by stacking the columns of $\mathrm{X}$ on top each other.

\section{System Model ANd PIC GRoup DeCoding}

In this section, we first briefly describe the system model and then describe the PIC group decoding proposed in [38].

\section{A. System Model}

We consider a MIMO transmission with $M$ transmit antennas and $N$ receive antennas over block fading channels. The received signal matrix $\mathbf{Y} \in \mathbb{C}^{T \times N}$ is

$$
\mathbf{Y}=\sqrt{\frac{\rho}{\mu}} \mathbf{X H}+\mathbf{W}
$$


where $\mathbf{X} \in \mathbb{C}^{T \times M}$ is the codword matrix, transmitted over $T$ time slots, $\mathbf{W} \in \mathbb{C}^{T \times N}$ is a noise matrix with independent and identically distributed (i.i.d.) entries being circularly symmetric complex Gaussian distributed $\mathcal{C N}(0,1), \mathbf{H} \in \mathbb{C}^{M \times N}$ is the channel matrix whose entries are also i.i.d. with the distribution $\mathcal{C N}(0,1), \rho$ denotes the average signal-to-noise ratio (SNR) per receive antenna and $\mu$ is the normalization factor to ensure that the average energy of the coded symbols transmitting from all antennas during one symbol period is 1 . The realization of $\mathbf{H}$ is assumed to be known at the receiver, but not known at the transmitter. Therefore, the signal power is allocated uniformly across the transmit antennas.

Definition 1 (Code Rate): Let $L$ be the number of independent information symbols $\left\{s_{l}\right\}, l=$ $1, \cdots, L$ per codeword $\mathrm{X}$, selected from a complex constellation $\mathcal{A}$. The code rate of the STBC is defined as $R=\frac{L}{T}$ symbols per channel use. If $L=T M$, the STBC is said to have full rate, i.e., $R=M$ symbols per channel use.

In this paper, we consider that information symbols $\left\{s_{l}\right\}, l=1, \cdots, L$ are coded by linear dispersion STBC as

$$
\mathbf{X}=\sum_{l=1}^{L} \mathbf{A}_{l} s_{l},
$$

where $\mathbf{A}_{l} \in \mathbb{C}^{T \times M}$ is the linear STBC matrix.

To decode the transmitted sequence $\mathbf{s}$ at the receiver, we need to extract $\mathbf{s}$ from $\mathbf{X}$. This can be done by as follows. By substituting (2) into (1), we get

$$
\mathbf{Y}=\sqrt{\frac{\rho}{\mu}} \sum_{l=1}^{L} \mathbf{A}_{l} \mathbf{H} s_{l}+\mathbf{W} .
$$

Then, by taking vectorization of the matrix $\mathrm{Y}$ we have

$$
\begin{aligned}
\mathbf{y} \triangleq \operatorname{vec}(\mathbf{Y}) & =\sqrt{\frac{\rho}{\mu}} \sum_{l=1}^{L} \operatorname{vec}\left(\mathbf{A}_{l} \mathbf{H}\right) s_{l}+\operatorname{vec}(\mathbf{W}) \\
& =\sqrt{\frac{\rho}{\mu}} \mathcal{H} \mathbf{s}+\mathbf{w}
\end{aligned}
$$

where $\mathbf{y} \in \mathbb{C}^{T N \times 1}, \mathbf{w} \in \mathbb{C}^{T N \times 1}, \mathbf{s}=\left[\begin{array}{llll}s_{1} & s_{2} & \cdots & s_{L}\end{array}\right]^{t}$, and $\mathcal{H} \in \mathbb{C}^{T N \times L}$ is an equivalent channel matrix,

$$
\mathcal{H}=\left[\begin{array}{llll}
\mathbf{g}_{1} & \mathbf{g}_{2} & \cdots & \mathbf{g}_{L}
\end{array}\right]
$$


with the $l$-th column $\mathbf{g}_{l}=\operatorname{vec}\left(\mathbf{A}_{l} \mathbf{H}\right), l=1,2, \cdots, L$.

For a $\mathrm{ZF}$ receiver, the estimate $\hat{\mathbf{s}}^{\mathrm{ZF}}$ of the transmitted symbol sequence $\mathbf{s}$ is,

$$
\hat{\mathbf{s}}^{\mathrm{ZF}}=\arg \min _{\mathbf{s} \in \mathcal{A}^{L}}\left\|\mathbf{Q}^{\mathrm{ZF}} \mathbf{y}-\mathbf{s}\right\|^{2},
$$

where $\mathrm{Q}^{\mathrm{ZF}}=\sqrt{\frac{\mu}{\rho}}\left(\mathcal{H}^{H} \mathcal{H}\right)^{-1} \mathcal{H}^{H}$. Equivalently, it can be written as the single-symbol decoding as follows,

$$
\hat{\mathbf{s}}_{l}^{\mathrm{ZF}}=\arg \min _{s_{l} \in \mathcal{A}}\left\|\left[\mathbf{Q}^{\mathrm{ZF}}\right]_{l,:} \mathbf{y}-s_{l}\right\|^{2}, \quad l=1,2 \cdots, L,
$$

where $\left[\mathrm{Q}^{\mathrm{ZF}}\right]_{l,:}$ denotes the $l$-th row of $\mathrm{Q}^{\mathrm{ZF}}$.

For an ML receiver, the estimate of $\hat{\mathbf{s}}^{\mathrm{ML}}$ that achieves the minimum of the squared Frobenius norm is given by

$$
\hat{\mathbf{s}}^{\mathrm{ML}}=\arg \min _{\mathbf{s} \in \mathcal{A}^{L}}\left\|\mathbf{y}-\sqrt{\frac{\rho}{\mu}} \mathcal{H} \mathbf{s}\right\|^{2} .
$$

In the ML decoding, computations of squared Frobenius norms for all possible codewords are needed and therefore result in prohibitively huge computational complexity when the length of the information symbols vector to be decoded is large. In the following, we give a metric to evaluate the computational complexity of the ML decoding, which is the same as the one shown in [31, Definition 2].

Definition 2 (Decoding Complexity): The decoding complexity $\mathcal{O}$ is defined as the number of squared Frobenius norms $\|\cdot\|^{2}$ that should be computed in the decoding process.

With the above definition, we have the following two remarks.

Remark 1: The decoding complexity of the ZF detection is $\mathcal{O}=L \cdot|\mathcal{A}|$, i.e., $L$ times of the cardinality of the signal constellation. It is equivalent to the single-symbol decoding complexity.

Remark 2: The decoding complexity of the ML detection is $\mathcal{O}=|\mathcal{A}|^{L}$, i.e., the complexity of the full exhaustive search of all $L$ information symbols drawn from the constellation $\mathcal{A}$.

We next describe the PIC group decoding studied in [38].

\section{B. PIC Group Decoding}

Define index set $\mathcal{I}$ as

$$
\mathcal{I}=\{1,2, \cdots, L\}
$$


where $L$ is the number of information symbols in s. We then partition $\mathcal{I}$ into $P$ groups: $\mathcal{I}_{1}, \mathcal{I}_{2}, \cdots, \mathcal{I}_{P}$ with

$$
\mathcal{I}_{p}=\left\{I_{p, 1}, I_{p, 2}, \cdots, I_{p, l_{p}}\right\}, \quad p=1,2, \cdots, P
$$

where $l_{p}$ is the cardinality of the subset $\mathcal{I}_{p}$. We call $\mathcal{I}=\left\{\mathcal{I}_{1}, \mathcal{I}_{2}, \cdots, \mathcal{I}_{P}\right\}$ a grouping scheme. For such a grouping scheme, we have

$$
\mathcal{I}=\bigcup_{p=1}^{P} \mathcal{I}_{p}, \text { and } \sum_{p=1}^{P} l_{p}=L
$$

Define

$$
\begin{aligned}
\mathbf{s}_{p} & =\left[\begin{array}{llll}
s_{I_{p, 1}} & s_{I_{p, 2}} & \cdots & s_{I_{p, l_{p}}}
\end{array}\right]^{t}, p=1, \cdots, P . \\
\mathbf{G}_{p} & =\left[\begin{array}{llll}
\mathbf{g}_{I_{p, 1}} & \mathbf{g}_{I_{p, 2}} & \cdots & \mathbf{g}_{I_{p, l_{p}}}
\end{array}\right], p=1, \cdots, P .
\end{aligned}
$$

With these notations, (4) can be written as

$$
\mathbf{y}=\sqrt{\frac{\rho}{\mu}} \sum_{p=1}^{P} \mathbf{G}_{p} \mathbf{s}_{p}+\mathbf{w} .
$$

Suppose we want to decode the symbols embedded in the group $\mathbf{s}_{p}$. The PIC group decoding first implements linear interference cancellation with a suitable choice of matrix $\mathbf{Q}_{p}$ in order to completely eliminate the interferences from other groups [38], i.e., $\mathbf{Q}_{p} \mathbf{G}_{q}=\mathbf{0}, \forall q \neq p$ and $q=1,2, \cdots, P$. Then, we have

$$
\begin{aligned}
\mathbf{z}_{p} & \triangleq \mathbf{Q}_{p} \mathbf{y} \\
& =\sqrt{\frac{\rho}{\mu}} \mathbf{Q}_{p} \mathbf{G}_{p} \mathbf{s}_{p}+\mathbf{Q}_{p} \mathbf{w}, \quad p=1,2, \cdots, P,
\end{aligned}
$$

where the interference cancellation matrix $\mathbf{Q}_{p}$ can be chosen as follows [38],

$$
\mathbf{Q}_{p}=\mathbf{I}_{T N}-\mathbf{G}_{p}^{c}\left(\left(\mathbf{G}_{p}^{c}\right)^{H} \mathbf{G}_{p}^{c}\right)^{-1}\left(\mathbf{G}_{p}^{c}\right)^{H}, \quad p=1,2, \cdots, P
$$

in case

$$
\mathbf{G}_{p}^{c}=\left[\begin{array}{llllll}
\mathbf{G}_{1} & \cdots & \mathbf{G}_{p-1} & \mathbf{G}_{p+1} & \cdots & \mathbf{G}_{P}
\end{array}\right]
$$

has full column rank. If $\mathrm{G}_{p}^{c}$ does not have full column rank, then we need to pick a maximal linear independent vector group from $\mathbf{G}_{p}^{c}$ and in this case a projection matrix $\mathbf{Q}_{p}$ can be found too [38]. 
Afterwards, the symbols in the group $\mathbf{s}_{p}$ are decoded with the ML decoding algorithm as follows,

$$
\hat{\mathbf{s}}_{p}=\arg \min _{\mathbf{s}_{p} \in \mathcal{A}^{l_{p}}}\left\|\mathbf{z}_{p}-\sqrt{\frac{\rho}{\mu}} \mathbf{Q}_{p} \mathbf{G}_{p} \mathbf{s}_{p}\right\|^{2} .
$$

The above PIC group decoding is connected to some of the known decodings as in the following remarks.

Remark 3 (ML and PIC Group Decoding): For one special case of $P=1$, the grouping scheme is $\mathcal{I}=\left\{\mathcal{I}_{1}\right\}$ with $\mathcal{I}_{1}=\mathcal{I}$. From (13), we have $\mathbf{Q}_{p}=\mathbf{I}_{T N}$. Then, the PIC group decoding is equivalent to the ML decoding where all information symbols are jointly decoded.

Remark 4 (ZF and PIC Group Decoding): For the special case of $P=L$, the grouping scheme is $\mathcal{I}=\left\{\mathcal{I}_{1}, \mathcal{I}_{2}, \cdots, \mathcal{I}_{L}\right\}=\{\{1\},\{2\}, \cdots,\{L\}\}$, i.e., every single symbol is regarded as one group. Then, the PIC group decoding is equivalent to the ZF decoding where every single symbol is separated from all the other symbols and then decoded.

Remark 5 (ZF, ML and PIC Group Decoding): The PIC group decoding with $1 \leq P \leq L$ can be viewed as an intermediate decoding approach between the ML decoding and the ZF decoding. Alternatively, the ML decoding and the ZF decoding can both be regarded as the special cases of the PIC group decoding corresponding to $P=1$ and $P=L$, respectively.

Remark 6 (PIC Group Decoding Complexity): For the PIC group decoding, the following two steps are needed: the group zero-forcing to cancel the interferences coming from all the other groups as shown in (12) and the group ML decoding to jointly decode the symbols in one group as shown in (15). Therefore, the decoding complexity of the PIC group decoding should reside in the above two steps. Note that the interference cancellation process shown in (12) mainly involves with linear matrix computations, whose computational complexity is small compared to the ML decoding for an exhaustive search of all candidate symbols. Therefore, to evaluate the decoding complexity of the PIC group decoding, we mainly focus on the computational complexity of the ML decoding within the PIC group decoding algorithm. According to Definition 2, the ML decoding complexity in the PIC group decoding algorithm is $\mathcal{O}=\sum_{p=1}^{P}|\mathcal{A}|^{l_{p}}$. It can be seen that the PIC group decoding provides a flexible decoding complexity which can be from the ZF decoding complexity $L|\mathcal{A}|$ to the ML decoding complexity $|\mathcal{A}|^{L}$. 
Remark 7 (PIC-SIC Group Decoding): In [38], an SIC-aided PIC group decoding algorithm, namely PIC-SIC group decoding was proposed. Similar to the BLAST detection algorithm [41], the PIC-SIC group decoding is performed after removing the already-decoded symbol set from the received signals to reduce the interference. If each group has only one symbol, then the PIC-SIC group will be equivalent to the BLAST detection.

\section{STBC Design Criterion with PIC Group Decoding}

The performance of a decoding algorithm for a wireless communication system is related to the diversity order. If the average probability of a detection error for communication over a fading channel usually behaves as:

$$
P_{e}(\mathrm{SNR}) \leq c \cdot \mathrm{SNR}^{-G_{d}}
$$

where $c$ is a constant and $G_{d}$ is called the diversity order of the system. For an MIMO communication system, the maximum diversity order is $M N$, i.e., the product of the number of transmit antennas and the number of receiver antennas. In order to optimize the reception performance of the MIMO system, a full diversity is usually pursued which can be achieved by a proper signal transmission scheme or data format (e.g., STBC). In [2], the "rank-and-determinant criterion" of STBC design was proposed to maximize both the diversity gain $G_{d}$ and the coding gain $\frac{1}{c}$ of the MIMO system with an ML decoding. Recently, in [38] an STBC design criterion was derived to achieve full diversity when the PIC group decoding is used at the receiver. In the following, we cite the main result of the STBC design criterion proposed in [38].

\section{Proposition 1: [38, Theorem 1] [Full-Diversity Criterion under PIC Group Decoding]}

For an STBC X with the PIC group decoding, the full diversity is achieved when

1) the code $\mathbf{X}$ satisfies the full rank criterion, i.e., it achieves full diversity when the ML receiver is used; and

2) $\mathbf{G}_{1}, \mathbf{G}_{2}, \cdots, \mathbf{G}_{P}$ are linearly independent vector groups for any $\mathbf{H} \neq \mathbf{0 .}$

In [38], the STBC achieving full diversity with PIC group decoding were proposed for 2 and 4 transmit antennas. However, a systematic code design of the full-diversity STBC with PIC group decoding remains an open problem.

Proposition 2: [38, p.4374] [Full-Diversity Criterion under PIC-SIC Group Decoding] 
For an STBC X with the PIC-SIC group decoding, the full diversity is achieved when

1) the code $X$ satisfies the full rank criterion, i.e., it achieves full diversity when the ML receiver is used; and

2) at each decoding stage, $\mathbf{G}_{q_{1}}$, which corresponds to the current to-be decoded symbol group $\mathbf{s}_{q_{1}}$, the remaining groups $\mathbf{G}_{q_{2}}, \cdots, \mathbf{G}_{q_{L}}$ corresponding to yet uncoded symbol groups are linearly independent vector groups for any $\mathbf{H} \neq \mathbf{0}$.

\section{A Design of STBC WITH PIC GRoup DeCODING}

In this section, we first propose a systematic design of high-rate STBC which has a rate up to $M$ symbols per channel use and achieves full diversity with the ML decoding. The systematic design of the STBC is structured with multiple diagonal layers. Then, we prove that the proposed STBC with two diagonal layers can obtain full diversity with the PIC group decoding and the code rate can be up to 2 symbols per channel use. Finally, we prove that the proposed STBC with any number of diagonal layers can obtain full diversity with PIC-SIC group decoding and the code rate can be up to $M$ symbols per channel use.

\section{A. Encoding Technique}

Our proposed space-time code $\mathbf{C}$, i.e., $\mathbf{X}$ in (1), is of size $T \times M$ (for any given $T, M$ and $T \geq M)$ and will be transmitted from $M$ antennas over $T$ time slots. Let $P=T-M+1$. The symbol stream $\left\{s_{l}\right\}, l=1, \cdots, L$ (composed of $L=M P$ complex symbols chosen from QAM constellation and then scaled by $1 / \sqrt{E\left[\left|s_{l}\right|^{2}\right]}$ ) is first parsed into $M \times 1$ symbol vectors $\mathbf{s}_{p}$ $(p=1,2, \cdots, P)$. Each symbol vector is linearly precoded by an $M \times M$ matrix $\boldsymbol{\Theta}$, which is a chosen constellation rotation matrix. Next, the $M \times 1$ vector $\Theta \mathbf{s}_{p}$ is used to form the space-time

code matrix $\mathbf{C}$, in which the $p$-th descending diagonal from left to right is the diagonal form of $\Theta \mathbf{s}_{p}$. 
The resulting transmitted code matrix $\mathrm{C}$ is given by

$$
\mathbf{C}=\left[\begin{array}{cccc}
X_{1,1} & 0 & \cdots & 0 \\
X_{2,1} & X_{1,2} & \ddots & \vdots \\
\vdots & X_{2,2} & \ddots & 0 \\
X_{P, 1} & \vdots & \ddots & X_{1, M} \\
0 & X_{P, 2} & \ddots & X_{2, M} \\
\vdots & 0 & \ddots & \vdots \\
0 & \vdots & \ddots & X_{P, M}
\end{array}\right]
$$

where the $p$-th descending diagonal from left to right, denoted by $\mathbf{X}_{p}=\left[\begin{array}{llll}X_{p, 1} & X_{p, 2} & \cdots & X_{p, M}\end{array}\right]^{t}$ is given by

$$
\mathbf{X}_{p}=\Theta_{\mathbf{s}_{p}}, \quad p=1,2, \cdots, P
$$

and the $M \times 1$ information symbol vector $\mathbf{s}_{p}$ is given by

$$
\mathbf{s}_{p}=\left[\begin{array}{llll}
s_{(p-1) M+1} & s_{(p-1) M+2} & \cdots & s_{p M}
\end{array}\right]^{t},
$$

for $\quad p=1,2, \cdots, P$.

Proposition 3: The proposed STBC in (16) has asymptotically full rate when the block length is sufficiently large.

Proof: In the codeword in (16), a total number of $M P$ independent information symbols are encoded into the codeword $\mathbf{C}$, which is then transmitted from $M$ antennas over $T$ time slots. The code rate of transmission is therefore

$$
R=\frac{M P}{T}=\frac{M(T-M+1)}{T}=M\left(1-\frac{M-1}{T}\right) .
$$

For a very large block length $T$, it can be seen that the rate $R$ of the proposed ST coding scheme approaches $M$ symbols per channel use, i.e. the full rate.

\section{B. Choice of Rotation Matrix $\Theta$}

In [40], the rotation matrix $\Theta$ was designed for diagonal STBC to achieve the full diversity gain and the optimal diversity product. With the optimal cyclotomic lattices design for $M$ transmit 
antennas, from [40, Table I] we can get a set of integers $(m, n)$ and let $K=m n$. Then, the optimal lattice $\Theta$ is given by [40, Eq. (16)]

$$
\boldsymbol{\Theta}=\left[\begin{array}{llll}
\zeta_{K} & \zeta_{K}^{2} & \cdots & \zeta_{K}^{M} \\
\zeta_{K}^{1+n_{2} m} & \zeta_{K}^{2\left(1+n_{2} m\right)} & \cdots & \zeta_{K}^{M\left(1+n_{2} m\right)} \\
\vdots & \vdots & \ddots & \vdots \\
\zeta_{K}^{1+n_{M} m} & \zeta_{K}^{2\left(1+n_{M} m\right)} & \cdots & \zeta_{K}^{M\left(1+n_{M} m\right)}
\end{array}\right] .
$$

where $\zeta_{K}=\exp (\mathbf{j} 2 \pi / K)$ with $\mathbf{j}=\sqrt{-1}$ and $n_{2}, n_{3}, \cdots, n_{M}$ are distinct integers such that $1+n_{i} m$ and $K$ are co-prime for any $2 \leq i \leq M$.

Example 1: For 4 transmit antennas we can choose $m=3, n=5$ and $K=15$ according to [40, Table I]. Then, in order to ensure that $1+n_{i} m$ and $K$ are co-prime for any $2 \leq i \leq M$ we can obtain $n_{2}=1, n_{3}=2, n_{4}=4$. When $m=3$, the signal constellation is located on the equal literal triangular lattice. When $m=4, n$ can be 4 and $n_{i}$ can be $0,1,2,3$, and in this case the signal constellation is located on the square lattice.

Example 2: For 5 transmit antennas we can select $m=n=5$ and $K=25$. Then, $n_{2}=1$, $n_{3}=2, n_{4}=3, n_{5}=4$.

The cyclotomic design of the matrix $\Theta$ is vital for the design of the algebraic STBC. In the following, we show some properties of the matrix $\Theta$ that will be used later for our design.

Property 1: [40] The diagonal cyclotomic ST code $\Omega$ defined by $\Omega=\left\{\operatorname{diag}\left[\begin{array}{llll}X_{1} & X_{2} & \cdots & X_{M}\end{array}\right]\right\}$ achieves full diversity under ML decoding, where $\left[\begin{array}{llll}X_{1} & X_{2} & \cdots & X_{M}\end{array}\right]^{t}=\Theta\left[\begin{array}{llll}s_{1} & s_{2} & \cdots & s_{M}\end{array}\right]^{t^{\prime}}$ and $\Theta$ is given by (20).

Property 2: Every entry of the matrix $\Theta$ in (20) is non-zero.

This property is obvious from (20).

\section{Achieving Full Diversity with ML Decoding}

We show the main result of the proposed STBC when an ML decoding is used at the receiver, as follows.

Theorem 1 (Full Diversity with ML Decoding): Consider a MIMO transmission with $M$ transmit antennas and $N$ receive antennas over block fading channels. The STBC $\mathrm{C}$ as described in 
(16) achieves full diversity under the ML decoding.

Proof of Theorem 1: In order to prove that the ST code C in (16) can obtain full diversity under ML decoding, it is sufficient to prove that $\Delta_{\mathbf{C}}=\mathbf{C}-\hat{\mathbf{C}}$ achieves full rank for any distinct pair of ST codewords $\mathbf{C}$ and $\hat{\mathbf{C}}$.

For any pair of distinct codewords $\mathbf{C}$ and $\hat{\mathbf{C}}$, there exists at least one index $p(1 \leq p \leq P)$ such that $\mathbf{X}_{p}-\hat{\mathbf{X}}_{p} \neq \mathbf{0}$, where $\mathbf{X}_{p}$ and $\hat{\mathbf{X}}_{p}$ are related to $\mathbf{s}_{p}$ and $\hat{\mathbf{s}}_{p}$ from (17), respectively. Let $p$ denote the minimum index of vectors satisfying $\mathbf{X}_{p}-\hat{\mathbf{X}}_{p} \neq \mathbf{0}$. Then, for any index $q$ with $q<p$, it must have $\mathbf{X}_{q}-\hat{\mathbf{X}}_{q}=\mathbf{0}$. Define $\breve{X}=X-\hat{X}$ as the difference between symbols $X$ and $\hat{X}$. Then, from (16) $\Delta_{\mathbf{C}}$ can be expressed as

$$
\Delta_{\mathbf{C}}=\left[\begin{array}{cccc}
0 & 0 & \cdots & 0 \\
\vdots & \vdots & \ddots & \vdots \\
0 & \vdots & \ddots & \vdots \\
\breve{X}_{p, 1} & 0 & \ddots & \vdots \\
\vdots & \breve{X}_{p, 2} & \ddots & \vdots \\
\breve{X}_{P, 1} & \vdots & \ddots & 0 \\
0 & \breve{X}_{P, 2} & \ddots & \breve{X}_{p, M} \\
\vdots & 0 & \ddots & \vdots \\
0 & \vdots & \ddots & \breve{X}_{P, M}
\end{array}\right]
$$

where $\breve{X}_{p, m} \neq 0$ for $m=1,2, \cdots, M$. This is because for $\mathbf{X}_{p}-\hat{\mathbf{X}}_{p} \neq \mathbf{0}$, it exists $\mathbf{s}_{p}-\hat{\mathbf{s}}_{p} \neq \mathbf{0}$. Due to the suitably chosen constellation rotation matrix $\Theta$ in (20), $\mathbf{X}_{p}-\hat{\mathbf{X}}_{p}$ must have nonzero entries for any $\mathbf{s}_{p} \neq \hat{\mathbf{s}}_{p}$. Then, the matrix $\Delta_{\mathbf{C}}$ has full rank.

The full rankness of $\Delta_{\mathbf{C}}$ can be examined similar to that for the Toeplitz code (or delay diversity code) [33] by checking if the columns of $\Delta_{\mathbf{C}}$ are linearly independent. Specifically, we establish $\Delta_{\mathbf{C}} \bar{\alpha}=\mathbf{0}$ with $\bar{\alpha}=\left[\begin{array}{llll}\alpha_{1} & \alpha_{2} & \cdots & \alpha_{M}\end{array}\right]^{t}$. First, we examine the $p$-th equation in $\Delta_{\mathbf{C}} \bar{\alpha}=\mathbf{0}$ and get $\alpha_{1} \breve{X}_{p, 1}=0$. Because $\breve{X}_{p, m} \neq 0$ for $m=1,2, \cdots, M, \alpha_{1}=0$. Then, we examine the $(p+1)$-th equation and get $\alpha_{2} \breve{X}_{p, 2}=0$. Immediately, $\alpha_{2}=0$. Likewise, we examine the $(p+2)$-th equation until $(p+M-1)$-th equation, and we can get $\alpha_{1}=\alpha_{2}=\cdots=\alpha_{M}=0$. Therefore, all columns of $\Delta_{\mathbf{C}}$ are linearly independent and $\Delta_{\mathbf{C}}$ has full rank.

This property will be used in next section in the proof of the full diversity property under the PIC group decoding. 
D. Achieving Full Diversity with PIC Group Decoding when $P=2$

We show the main result of the proposed STBC when a PIC group decoding with a particular grouping scheme is used at the receiver, as follows.

Theorem 2 (Full Diversity with PIC Group Decoding when $P=2$ ): Consider a MIMO transmission with $M$ transmit antennas and $N$ receive antennas over block fading channels. The STBC $\mathrm{C}$ as described in (16) with two diagonal layers (i.e., $P=2$ ) is used at the transmitter. The equivalent channel matrix is $\mathcal{H} \in \mathbb{C}^{T N \times M P}$. If the received signal is decoded using the PIC group decoding with the grouping scheme $\mathcal{I}=\left\{\mathcal{I}_{1}, \mathcal{I}_{2}\right\}$ where $\mathcal{I}_{p}=\{(p-1) M+1,(p-1) M+$ $2, \cdots, p M\}$ for $p=1,2$, i.e., the size of each group is equal to the number of transmit antennas $M$, then the code $\mathrm{C}$ achieves the full diversity. The code rate of the full-diversity STBC can be up to 2 symbols per channel use.

In order to prove Theorem 2, let us first introduce the following lemmas.

Lemma 1: Consider the system as described in Theorem 2 with $N=1$ and the STBC C as given by (16),

1) the equivalent channel matrix $\mathcal{H} \in \mathbb{C}^{T \times M P}$ can be expressed as

$$
\mathcal{H}=\left[\begin{array}{llll}
\mathbf{G}_{1} & \mathbf{G}_{2} & \cdots & \mathbf{G}_{P}
\end{array}\right]
$$

where

$$
\mathbf{G}_{p}=\left[\begin{array}{c}
\mathbf{0}_{(p-1) \times M} \\
\operatorname{diag}(\mathbf{h}) \boldsymbol{\Theta} \\
\mathbf{0}_{(P-p) \times M}
\end{array}\right], \quad p=1,2, \cdots, P,
$$

2) When $P=2, \mathbf{G}_{1}$ and $\mathbf{G}_{2}$ are linearly independent vector groups as long as $\mathbf{h} \neq \mathbf{0}$, where $\mathbf{h}=\mathbf{H}$.

A proof of Lemma 1 is given in Appendix I.

Lemma 2: Consider the system as described in Theorem 2 and the STBC C as given by (16). For the equivalent channel matrix $\mathcal{H} \in \mathbb{C}^{T N \times M P}, \mathbf{G}_{1}, \mathbf{G}_{2}, \cdots, \mathbf{G}_{P}$ are linearly independent vector groups for $\mathbf{h} \neq \mathbf{0}$ when $N>1$ if and only if $\mathbf{G}_{1}, \mathbf{G}_{2}, \cdots, \mathbf{G}_{P}$ are linearly independent vector groups for $\mathbf{h} \neq \mathbf{0}$ when $N=1$. 
The proof of Lemma 2 is straightforward and is also the same as what is mentioned in [38].

Proof of Theorem 2: As shown in Proposition 1 , a codeword C with PIC group decoding can obtain the full diversity if

1) $\mathrm{C}$ achieves the full diversity with the ML receiver, and

2) $\mathbf{G}_{1}, \mathbf{G}_{2}, \cdots, \mathbf{G}_{P}$ are linearly independent vector groups as long as $\mathbf{h} \neq \mathbf{0}$.

For the proposed code $\mathrm{C}$ in (16) with $P=2$, the first condition is satisfied as shown in Theorem 1. The second condition is satisfied as shown in Lemma 1 for $N=1$ and Lemma 2 for $N>1$, respectively. Therefore, the code $\mathrm{C}$ in (16) with $P=2$ can obtain full diversity with the PIC group decoding provided that the grouping scheme is $\mathcal{I}=\left\{\mathcal{I}_{1}, \mathcal{I}_{2}\right\}$ where $\mathcal{I}_{p}=$ $\{(p-1) M+1,(p-1) M+2, \cdots, p M\}$ for $p=1,2$.

The code rate of the full-diversity STBC with the PIC group decoding can be derived by substituting $P=2$ and $T=M+1$ into (19) as

$$
R=\frac{2 M}{M+1} .
$$

For a large number of transmit antennas, the rate approaches to (but not larger than) 2 symbols per channel use.

Corollary 1: The decoding complexity of the PIC group decoding of the proposed STBC with the grouping scheme as described in Theorem 2 is $\mathcal{O}=P \cdot|\mathcal{A}|^{M}$.

Remark 8: The decoding complexity of the proposed STBC with the PIC group decoding is equivalent to the ML decoding of $M$ independent information symbols jointly. As shown in (19), the code rate of the proposed STBC in (16) for a given $M$ can be increased by embedding larger number of groups in the codeword, i.e., increasing the value of $P$. It is noteworthy to mention that the increase of the code rate does not result in the increase of the decoding complexity.

\section{E. Achieving Full Diversity with PIC-SIC Group Decoding}

For the proposed STBC with any number of layers and the PIC-SIC group decoding we have the following results.

Theorem 3 (Full Diversity with PIC-SIC Group Decoding): Consider a MIMO transmission with $M$ transmit antennas and $N$ receive antennas over block fading channels. The STBC C 
as described in (16) with $P$ diagonal layers is used at the transmitter. The equivalent channel matrix is $\mathcal{H} \in \mathbb{C}^{T N \times M P}$. If the received signal is decoded using the PIC-SIC group decoding with the sequential order and with the grouping scheme being $\mathcal{I}=\left\{\mathcal{I}_{1}, \cdots, \mathcal{I}_{P}\right\}$ where $\mathcal{I}_{p}=$ $\{(p-1) M+1,(p-1) M+2, \cdots, p M\}$ for $p=1, \cdots, P$, i.e., the size of each group is equal to the number of transmit antennas $M$, then the code $\mathrm{C}$ achieves the full diversity. The code rate of the full-diversity STBC can be up to $M$ symbols per channel use.

The proof of this theorem is simple. Observing that $\mathcal{H}=\left[\begin{array}{lll}\mathbf{G}_{1} & \cdots & \mathbf{G}_{P}\end{array}\right]$ and the group $\mathbf{G}_{p}$ is linearly independent from the groups $\left\{\mathbf{G}_{p+1}, \cdots, \mathbf{G}_{P}\right\}$ for any $p$ and $1 \leq p \leq P-1$ where $\mathbf{G}_{p}$ is given by (23), according to Proposition 2 the full diversity can be easily proved. The detailed proof is omitted.

\section{Code Design Examples}

In this section, we show a few code design examples. We denote $\mathbf{C}_{M, T, P}$ the code constructed by (16) for given parameters: $M$ the number of transmit antennas, $T$ the block length of the code, and $P$ the number of groups to be decoded in the PIC group decoding. For notational brevity, we only show the equivalent channel of the proposed codes for MISO systems.

\section{A. For Two Transmit Antennas}

Consider a code for 2 transmit antennas with 3 time slots. According to the code structure (16), we have

$$
\mathbf{C}_{2,3,2}=\left[\begin{array}{cc}
X_{1,1} & \\
X_{2,1} & X_{1,2} \\
& X_{2,2}
\end{array}\right],
$$

where $\left[\begin{array}{ll}X_{1,1} & X_{1,2}\end{array}\right]^{t}=\Theta\left[\begin{array}{ll}s_{1} & s_{2}\end{array}\right]^{t}$ and $\left[\begin{array}{ll}X_{2,1} & X_{2,2}\end{array}\right]^{t}=\Theta\left[\begin{array}{ll}s_{3} & s_{4}\end{array}\right]^{t}$. The constellation rotation matrix $\Theta$ can be chosen as

$$
\boldsymbol{\Theta}=\left[\begin{array}{rr}
\gamma & \delta \\
-\delta & \gamma
\end{array}\right],
$$

where $\gamma=\cos \theta$ and $\delta=\sin \theta$ with $\theta=1.02$ [38].

The code rate of the code is $4 / 3$. In fact, this code is equivalent to the one proposed in [38, Section VI - Example 1]. 
The equivalent channel of the code $\mathbf{C}_{2,3,2}$ is given by

$$
\mathcal{H}=\left[\begin{array}{cccc}
\gamma h_{1} & \delta h_{1} & & \\
-\delta h_{2} & \gamma h_{2} & \gamma h_{1} & \delta h_{1} \\
& & -\delta h_{2} & \gamma h_{2}
\end{array}\right] .
$$

The grouping scheme for the PIC group decoding is $\mathcal{I}_{1}=\{1,2\}$ and $\mathcal{I}_{2}=\{3,4\}$. It can be seen that $G_{1}$ and $G_{2}$ are linearly independent. Then, the code can obtain full diversity with the PIC group decoding.

\section{B. For Four Transmit Antennas}

For given $T=5$, the code achieving full diversity with the PIC group decoding can be designed as follows,

$$
\mathbf{C}_{4,5,2}=\left[\begin{array}{cccc}
X_{1,1} & & & \\
X_{2,1} & X_{1,2} & & \\
& X_{2,2} & X_{1,3} & \\
& & X_{2,3} & X_{1,4} \\
& & & X_{2,4}
\end{array}\right] .
$$

This code has a code rate of $8 / 5$ and two groups to be decoded. The equivalent channel of the code $\mathbf{C}_{4,5,2}$ is

$$
\mathcal{H}=\left[\left[\begin{array}{c}
\mathrm{B} \\
\mathbf{0}_{1 \times 4}
\end{array}\right]\left[\begin{array}{c}
\mathbf{0}_{1 \times 4} \\
\mathbf{B}
\end{array}\right]\right],
$$

where $\mathbf{B}$ is given by

$$
\mathbf{B}=\left[\begin{array}{llll}
h_{1} \theta_{1,1} & h_{1} \theta_{1,2} & h_{1} \theta_{1,3} & h_{1} \theta_{1,4} \\
h_{2} \theta_{2,1} & h_{2} \theta_{2,2} & h_{2} \theta_{2,3} & h_{2} \theta_{2,4} \\
h_{3} \theta_{3,1} & h_{3} \theta_{3,2} & h_{3} \theta_{3,3} & h_{3} \theta_{3,4} \\
h_{4} \theta_{4,1} & h_{4} \theta_{4,2} & h_{4} \theta_{4,3} & h_{4} \theta_{4,4}
\end{array}\right]
$$

with $\theta_{i, j}$ being the $(i, j)$-th entry of the matrix $\Theta$ for $i, j=1,2,3,4$. The grouping scheme for the PIC group decoding is $\mathcal{I}_{1}=\{1,2,3,4\}$ and $\mathcal{I}_{2}=\{5,6,7,8\}$. It can be seen that the groups $\mathbf{G}_{1}$ and $\mathbf{G}_{2}$ are linearly independent to each other. Then, the code can obtain full diversity with the PIC group decoding. 
Consider $T=6$ time slots. We can get

$$
\mathbf{C}_{4,6,2}=\left[\begin{array}{cccc}
X_{1,1} & & & \\
0 & X_{1,2} & & \\
X_{2,1} & 0 & X_{1,3} & \\
& X_{2,2} & 0 & X_{1,4} \\
& & X_{2,3} & 0 \\
& & & X_{2,4}
\end{array}\right] .
$$

The code rate of the code $\mathbf{C}_{4,6,2}$ is $4 / 3$ which has the same rate as the one proposed in [38, Section VI - Example 2]. The equivalent channel of the code $\mathbf{C}_{4,6,2}$ is

$$
\mathcal{H}=\left[\left[\begin{array}{c}
\mathrm{B} \\
\mathbf{0}_{2 \times 4}
\end{array}\right]\left[\begin{array}{c}
\mathbf{0}_{2 \times 4} \\
\mathrm{~B}
\end{array}\right]\right],
$$

where $B$ is given by (28). Because the groups $G_{1}$ and $G_{2}$ are linearly independent to each other. Then, the code can obtain full diversity with the PIC group decoding.

Moreover, we can also design the code for $T=6$ with 3 layers (i.e., $P=3$ ) as follows,

$$
\mathbf{C}_{4,6,3}=\left[\begin{array}{cccc}
X_{1,1} & & & \\
X_{2,1} & X_{1,2} & & \\
X_{3,1} & X_{2,2} & X_{1,3} & \\
& X_{3,2} & X_{2,3} & X_{1,4} \\
& & X_{3,3} & X_{2,4} \\
& & & X_{3,4}
\end{array}\right] .
$$

The code rate of the code $\mathbf{C}_{4,6,3}$ is 2 and the equivalent channel is given by

$$
\mathcal{H}=\left[\begin{array}{lll}
\mathbf{G}_{1} & \mathbf{G}_{2} & \mathbf{G}_{3}
\end{array}\right],
$$

where

$$
\mathbf{G}_{1}=\left[\begin{array}{c}
\mathbf{B} \\
\mathbf{0}_{1 \times 4} \\
\mathbf{0}_{1 \times 4}
\end{array}\right], \quad \mathbf{G}_{2}=\left[\begin{array}{c}
\mathbf{0}_{1 \times 4} \\
\mathbf{B} \\
\mathbf{0}_{1 \times 4}
\end{array}\right], \quad \mathbf{G}_{3}=\left[\begin{array}{c}
\mathbf{0}_{1 \times 4} \\
\mathbf{0}_{1 \times 4} \\
\mathbf{B}
\end{array}\right] .
$$

It can be proved that the groups $\mathrm{G}_{1}, \mathrm{G}_{2}$, and $\mathrm{G}_{3}$ are not linearly independent groups. Therefore, according to Proposition 1 , the code $\mathrm{C}_{4,6,3}$ cannot achieve the full diversity with the PIC group decoding. 
However, the code $\mathbf{C}_{4,6,3}$ can obtain full diversity with PIC-SIC group decoding. This is because $G_{1}$ is linearly independent from $G_{2}$ and $G_{3}$, and $G_{2}$ is linearly independent from $G_{3}$. According to Proposition 2, with PIC-SIC group decoding and a proper decoding order $\{1,2,3\}$ or $\{3,2,1\}$, the code $\mathbf{C}_{4,6,3}$ can achieve the full diversity.

\section{For Five Transmit Antennas}

For given $T=6$ and $P=2$, the code is designed as follows,

$$
\mathbf{C}_{5,6,2}=\left[\begin{array}{ccccc}
X_{1,1} & & & & \\
X_{2,1} & X_{1,2} & & & \\
& X_{2,2} & X_{1,3} & & \\
& & X_{2,3} & X_{1,4} & \\
& & & X_{2,4} & X_{1,5} \\
& & & & X_{2,5}
\end{array}\right] .
$$

The code rate of the code $\mathbf{C}_{5,6,2}$ is $5 / 3$. The equivalent channel is

$$
\mathcal{H}=\left[\left[\begin{array}{c}
\operatorname{diag}(\mathbf{h}) \boldsymbol{\Theta}_{5} \\
\mathbf{0}_{1 \times 5}
\end{array}\right] \quad\left[\begin{array}{c}
\mathbf{0}_{1 \times 5} \\
\operatorname{diag}(\mathbf{h}) \boldsymbol{\Theta}_{5}
\end{array}\right]\right],
$$

where $\Theta_{5}$ is the rotation matrix of size $5 \times 5$.

The grouping scheme for the PIC group decoding of $\mathbf{C}_{5,6,2}$ is $\mathcal{I}_{1}=\{1,2,3,4,5\}$ and $\mathcal{I}_{2}=$ $\{6,7,8,9,10\}$. It can be seen that the groups $\mathbf{G}_{1}$ and $\mathbf{G}_{2}$ are linearly independent to each other. Then, the code $\mathbf{C}_{5,6,2}$ can obtain full diversity with the PIC group decoding.

\section{Another Design of STBC with PIC Group DeCoding}

Notice that the code design in (16) can only achieve the full diversity with PIC group decoding for two diagonal layers (r.f. Theorem 2) and the code rate is not larger than 2 symbols per channel use. With $P(P>2)$ diagonal layers in the code (16), the rate can be increased but the independence among $P$ channel groups $\left\{\begin{array}{lllll}\mathbf{G}_{1} & \mathbf{G}_{2} & \cdots & \mathbf{G}_{P}\end{array}\right\}$ in (22) is not satisfied, thereby may lose the full diversity gain. In this section, we propose a new code design which can achieve full diversity with PIC group decoding and a rate above 2. 


\section{A. Code Design}

For $M=3 p$ ( $p$ is an integer), our proposed STBC $\mathbf{C}_{M}$ for $M$ transmit antennas is given by

$$
\mathbf{C}_{3 p}=\left[\begin{array}{cccccccccc}
X_{1,1} & 0 & 0 & 0 & 0 & 0 & \cdots & 0 & 0 & 0 \\
0 & X_{2,2} & 0 & 0 & 0 & 0 & \ddots & 0 & 0 & 0 \\
0 & 0 & X_{3,3} & 0 & 0 & 0 & \ddots & 0 & 0 & 0 \\
X_{2,1} & 0 & 0 & X_{1,4} & 0 & 0 & \ddots & 0 & 0 & 0 \\
0 & X_{3,2} & 0 & 0 & X_{2,5} & 0 & \ddots & 0 & 0 & 0 \\
0 & 0 & X_{1,3} & 0 & 0 & X_{3,6} & \ddots & 0 & 0 & 0 \\
\ddots & \ddots & \ddots & \ddots & \ddots & \ddots & \ddots & \ddots & \ddots & \ddots \\
0 & 0 & 0 & 0 & 0 & 0 & \ddots & X_{1,3 p-2} & 0 & 0 \\
0 & 0 & 0 & 0 & 0 & 0 & \ddots & 0 & X_{2,3 p-1} & 0 \\
0 & 0 & 0 & 0 & 0 & 0 & \ddots & 0 & 0 & X_{3,3 p} \\
0 & X_{1,2} & 0 & 0 & 0 & 0 & \ddots & X_{2,3 p-2} & 0 & 0 \\
0 & 0 & X_{2,3} & 0 & 0 & 0 & \ddots & 0 & X_{3,3 p-1} & 0 \\
X_{3,1} & 0 & 0 & 0 & 0 & 0 & \ddots & 0 & 0 & X_{1,3 p} \\
0 & 0 & 0 & X_{3,4} & X_{1,5} & X_{2,6} & \ddots & 0 & 0 & 0 \\
\vdots & \vdots & \vdots & \vdots & \vdots & \vdots & \ddots & \vdots & \vdots & \vdots \\
0 & 0 & 0 & 0 & 0 & 0 & \ddots & X_{3,3 p-2} & X_{1,3 p-1} & X_{2,3 p}
\end{array}\right],
$$

where the symbol vector $\mathbf{X}_{i}=\left[X_{i, 1}, X_{i, 2}, \cdots, X_{i, M}\right]^{t}$ is given by

$$
\mathbf{X}_{i}=\mathbf{\Theta s}_{i}, i=1,2,3,
$$

$\Theta$ is an $M \times M$ constellation rotation matrix given by (20) and $\mathbf{s}_{i}=\left[s_{(i-1) M+1}, s_{(i-1) M+2}, \cdots, s_{i M}\right]^{t}$ is the $M \times 1$ information symbol vector. 
For $M=3 p-1$, our proposed $\mathrm{STBC} \mathbf{C}_{M}$ is given by

$$
\mathbf{C}_{3 p-1}=\left[\begin{array}{cccccccccccc}
X_{1,1} & 0 & 0 & 0 & 0 & 0 & \cdots & 0 & 0 & 0 & 0 & 0 \\
0 & X_{2,2} & 0 & 0 & 0 & 0 & \ddots & 0 & 0 & 0 & 0 & 0 \\
0 & 0 & X_{3,3} & 0 & 0 & 0 & \ddots & 0 & 0 & 0 & 0 & 0 \\
X_{2,1} & 0 & 0 & X_{1,4} & 0 & 0 & \ddots & 0 & 0 & 0 & 0 & 0 \\
0 & X_{3,2} & 0 & 0 & X_{2,5} & 0 & \ddots & 0 & 0 & 0 & 0 & 0 \\
0 & 0 & X_{1,3} & 0 & 0 & X_{3,6} & \ddots & 0 & 0 & 0 & 0 & 0 \\
\ddots & \ddots & \ddots & \ddots & \ddots & \ddots & \ddots & \ddots & \ddots & \ddots & \ddots & \ddots \\
0 & 0 & 0 & 0 & 0 & 0 & \ddots & X_{1,3 p-5} & 0 & 0 & 0 & 0 \\
0 & 0 & 0 & 0 & 0 & 0 & \ddots & 0 & X_{2,3 p-4} & 0 & 0 & 0 \\
0 & 0 & 0 & 0 & 0 & 0 & \ddots & 0 & 0 & X_{3,3 p-3} & 0 & 0 \\
0 & 0 & 0 & 0 & 0 & 0 & \ddots & X_{2,3 p-5} & 0 & 0 & X_{1,3 p-2} & 0 \\
0 & 0 & 0 & 0 & 0 & 0 & \ddots & 0 & 0 & X_{1,3 p-3} & X_{3,3 p-2} & 0 \\
0 & X_{1,2} & 0 & 0 & 0 & 0 & \ddots & 0 & 0 & 0 & X_{2,3 p-2} & 0 \\
0 & 0 & X_{2,3} & 0 & 0 & 0 & \ddots & 0 & 0 & 0 & 0 & X_{3,3 p-1} \\
X_{3,1} & 0 & 0 & 0 & 0 & 0 & \ddots & 0 & 0 & 0 & 0 & X_{1,3 p-1} \\
0 & 0 & 0 & X_{3,4} & X_{1,5} & X_{2,6} & \ddots & 0 & 0 & 0 & 0 & 0 \\
\vdots & \vdots & \vdots & \vdots & \vdots & \vdots & \ddots & \vdots & \vdots & \vdots & \vdots & \vdots \\
0 & 0 & 0 & 0 & 0 & 0 & \ddots & X_{3,3 p-5} & X_{1,3 p-4} & X_{2,3 p-3} & 0 & 0
\end{array}\right] .
$$


For $M=3 p+1$, our proposed $\mathrm{STBC} \mathbf{C}_{M}$ is given by

$$
\mathbf{C}_{3 p+1}=\left[\begin{array}{ccccccccccc}
X_{1,1} & 0 & 0 & 0 & 0 & 0 & \cdots & 0 & 0 & 0 & 0 \\
0 & X_{2,2} & 0 & 0 & 0 & 0 & \ddots & 0 & 0 & 0 & 0 \\
0 & 0 & X_{3,3} & 0 & 0 & 0 & \ddots & 0 & 0 & 0 & 0 \\
X_{2,1} & 0 & 0 & X_{1,4} & 0 & 0 & \ddots & 0 & 0 & 0 & 0 \\
0 & X_{3,2} & 0 & 0 & X_{2,5} & 0 & \ddots & 0 & 0 & 0 & 0 \\
0 & 0 & X_{1,3} & 0 & 0 & X_{3,6} & \ddots & 0 & 0 & 0 & 0 \\
\ddots & \ddots & \ddots & \ddots & \ddots & \ddots & \ddots & \ddots & \ddots & \ddots & \ddots \\
0 & 0 & 0 & 0 & 0 & 0 & \ddots & X_{1,3 p-2} & 0 & 0 & 0 \\
0 & 0 & 0 & 0 & 0 & 0 & \ddots & 0 & X_{2,3 p-1} & 0 & 0 \\
0 & 0 & 0 & 0 & 0 & 0 & \ddots & 0 & 0 & X_{3,3 p} & 0 \\
0 & 0 & 0 & 0 & 0 & 0 & \ddots & X_{2,3 p-2} & 0 & 0 & X_{1,3 p+1} \\
0 & 0 & 0 & 0 & 0 & 0 & \ddots & 0 & X_{3,3 p-1} & 0 & X_{2,3 p+1} \\
0 & 0 & 0 & 0 & 0 & 0 & \ddots & 0 & 0 & X_{1,3 p} & X_{3,3 p+1} \\
X_{3,1} & X_{1,2} & X_{2,3} & 0 & 0 & 0 & \ddots & 0 & 0 & 0 & 0 \\
0 & 0 & 0 & X_{3,4} & X_{1,5} & X_{2,6} & \ddots & 0 & 0 & 0 & 0 \\
\vdots & \vdots & \vdots & \vdots & \vdots & \vdots & \ddots & \vdots & \vdots & :: & \\
0 & 0 & 0 & 0 & 0 & 0 & \ddots & X_{3,3 p-2} & X_{1,3 p-1} & X_{2,3 p} & 0
\end{array}\right]
$$

Proposition 4: The proposed STBC has the rate as follows:

$$
R=\left\{\begin{array}{lll}
\frac{9 M}{4 M+6} & \text { for } & M=3 p \\
\frac{9 M}{4 M+4} & \text { for } & M=3 p-1 \\
\frac{9 M}{4 M+5} & \text { for } & M=3 p+1
\end{array}\right.
$$

Proof: For $M=3 p$, in the codeword (35) $3 M$ information symbols are sent over $M+3+$ $\frac{M}{3}-1$ time slots. Thus, the code rate is $\frac{9 M}{4 M+6}$. Similarly, it is easy to prove the code rate for cases of $M=3 p-1$ and $M=3 p+1$.

Remark 9 (Asymptotic Rate): It is obvious that the code rate (37) approaches to $9 / 4$ when a large number of transmit antennas are used. Its full diversity property will be proved in the next 
subsection. However, the full diversity code proposed by the first design in (16) cannot achieve a rate more than 2, which was shown in Theorem 2.

Remark 10 (Rate Comparison): Note that the code design in (16) can achieve full diversity with the PIC group decoding for $P=2$ only and the rate is $\frac{2 M}{M+1}$. In Table I, the comparison of the code rate between the first code design in (16) and the second design in (35)-(36) is given.

TABLE I

COMPARISON IN CODE RATE $R$ (SYMBOLS PER CHANNEL USE)

\begin{tabular}{|c|c|c|}
\hline & Code 16 & Code (35)-(36) \\
\hline & $P=2$ & $P=3$ \\
\hline$M=2$ & $4 / 3$ & $3 / 2$ \\
\hline$M=3$ & $3 / 2$ & $3 / 2$ \\
\hline$M=4$ & $8 / 5$ & $12 / 7$ \\
\hline$M=5$ & $5 / 3$ & $15 / 8$ \\
\hline$M=6$ & $12 / 7$ & $9 / 5$ \\
\hline$M=7$ & $7 / 4$ & $21 / 11$ \\
\hline$M=8$ & $16 / 9$ & 2 \\
\hline
\end{tabular}

Remark 11 (Decoding Complexity): The decoding complexity of the proposed STBC with the PIC group decoding is equivalent to the ML decoding of $M$ independent information symbols jointly. According to Definition 2, the ML decoding complexity in the PIC group decoding algorithm is $\mathcal{O}=3|\mathcal{A}|^{M}$.

\section{B. Full Diversity with PIC Group Decoding}

Next, we show that the proposed STBC in (35) achieves full diversity when a PIC group decoding is used at the receiver.

Theorem 4: Let the STBC C as described in (35) be used at the transmitter. There are $M$ transmit antennas and 1 receive antenna. If the received signal is decoded using the PIC group decoding with the grouping scheme $\mathcal{I}=\left\{\mathcal{I}_{1}, \mathcal{I}_{2}, \mathcal{I}_{3}\right\}$, where $\mathcal{I}_{i}=\{(i-1) M+1,(i-1) M+$ $2, \cdots, i M\}$ for $i=1,2,3$, then the code $\mathbf{C}$ achieves the full diversity.

In order to prove the Theorem 4, let us first introduce the following lemma. 
Lemma 3: Consider the system as described in Theorem 4 with $N=1$, and the channel matrix $\mathbf{H}=\left[\begin{array}{llll}h_{1} & h_{2} & \cdots & h_{M}\end{array}\right]^{t}$. Let $\mathbf{g}_{i}=h_{i} \boldsymbol{\Theta}_{i}$, where $\boldsymbol{\Theta}_{i}$ denotes the $i$ th row of the rotation matrix $\Theta$ for $i=1,2, \cdots, M$. Then, the equivalent channel matrix is given by

$$
\mathcal{H}=\left[\begin{array}{lll}
\mathbf{G}_{1} & \mathbf{G}_{2} & \mathbf{G}_{3}
\end{array}\right]=\left[\begin{array}{ccc}
\mathbf{g}_{1} & 0 & 0 \\
0 & \mathbf{g}_{2} & 0 \\
0 & 0 & \mathbf{g}_{3} \\
\mathbf{g}_{4} & \mathbf{g}_{1} & 0 \\
0 & \mathbf{g}_{5} & \mathbf{g}_{2} \\
\mathbf{g}_{3} & 0 & \mathbf{g}_{6} \\
\vdots & \vdots & \vdots \\
\mathbf{g}_{3 p-2} & \mathbf{g}_{3 p-5} & 0 \\
0 & \mathbf{g}_{3 p-1} & \mathbf{g}_{3 p-4} \\
\mathbf{g}_{3 p-3} & 0 & \mathbf{g}_{3 p} \\
\mathbf{g}_{2} & \mathbf{g}_{3 p-2} & 0 \\
0 & \mathbf{g}_{3} & \mathbf{g}_{3 p-1} \\
\mathbf{g}_{3 p} & 0 & \mathbf{g}_{1} \\
\mathbf{g}_{5} & \mathbf{g}_{6} & \mathbf{g}_{4} \\
\vdots & \vdots & \vdots \\
\mathbf{g}_{3 p-1} & \mathbf{g}_{3 p} & \mathbf{g}_{3 p-2}
\end{array}\right] .
$$

The proof of Lemma 3 is straightforward and it is easy to verify that $\mathbf{C H}=\mathcal{H}\left[\begin{array}{lll}\mathbf{s}_{1}^{t} & \mathbf{s}_{2}^{t} & \mathbf{s}_{3}^{t}\end{array}\right]^{t}$. Proof of Theorem 4:

Firstly, we show that the proposed STBC C in (35) achieves the full diversity with ML decoding, i.e., $\Delta \mathbf{C}=\mathbf{C}-\mathbf{C}^{\prime}$ achieves full rank for any distinct pair of codewords $\mathbf{X}$ and $\mathbf{X}^{\prime}$.

Since $\mathbf{X}$ and $\mathbf{X}^{\prime}$ are distinct, at least one pair of the information symbol vectors $\mathbf{s}_{i}$ and $\mathbf{s}_{i}^{\prime}$ are different. Suppose only one pair of the information symbol vectors are distinct, say $\mathbf{s}_{1}$ and $\mathbf{s}_{1}^{\prime}$. By the Property $1, \Delta X_{1, j}=X_{1, j}-X_{1, j}^{\prime} \neq 0$ for all $j=1,2, \cdots, M$. In this case, the matrix $\Delta \mathbf{C}$ is exactly a diagonal matrix with the rows rearranged. Thus, it achieves full rank.

If all three pairs of the information symbol vectors are distinct, then $\Delta X_{i, j}=X_{i, j}-X_{i, j}^{\prime} \neq 0$ for all $i=1,2,3$ and $j=1,2, \cdots, M$. It is easy to see from the codeword structure that $\Delta \mathbf{C}$ is exactly a lower triangular matrix with nonzero diagonal entries. Thus, it achieves full rank. 
If two pairs of the information symbol vectors are distinct and the other one pair of the information symbol vectors are the same, say $\mathbf{s}_{1}=\mathbf{s}_{1}^{\prime}$. After replacing the $(3 q-2)$ th row by the $(3 q+1)$ th row for $q=1,2, \cdots, \frac{M}{3}$, the matrix $\Delta \mathbf{C}$ becomes a lower triangular matrix with nonzero diagonal entries. Thus, it achieves full rank.

As shown above, $\Delta \mathbf{C}$ has full rank for all $\left[\begin{array}{lll}\mathbf{s}_{1} & \mathbf{s}_{2} & \mathbf{s}_{3}\end{array}\right] \neq\left[\begin{array}{lll}\mathbf{s}_{1}^{\prime} & \mathbf{s}_{2}^{\prime} & \mathbf{s}_{3}^{\prime}\end{array}\right]$, i.e., $\mathbf{X} \neq \mathbf{X}^{\prime}$.

Next, we show that $\mathbf{G}_{1}, \mathbf{G}_{2}$ and $\mathbf{G}_{3}$ are linearly independent vector groups as long as $\mathbf{H} \neq 0$. If $h_{1} \neq 0$, it is obvious that $\mathbf{G}_{1}$ is linearly independent of $\mathbf{G}_{2}$ and $\mathbf{G}_{3}$. Otherwise, $h_{1}=0$, if $h_{4} \neq 0, \mathbf{G}_{1}$ is also independent of $\mathbf{G}_{2}$ and $\mathbf{G}_{3}$. Therefore, we can reorder $\mathbf{H}$ as

$$
\left\{h_{1}, h_{4}, \cdots, h_{3 p-2}, h_{3 p}, h_{3 p-3}, \cdots, h_{3}, h_{2}, h_{5}, \cdots, h_{3 p-1}\right\} .
$$

Suppose $h_{j}$ is the first one in the sequence such that $h_{j} \neq 0$. According to the Property 2, the entries of $\mathbf{g}_{j}$ in $\mathbf{G}_{1}$ are not zeros, but the entries in the same row of $\mathbf{G}_{2}$ and $\mathbf{G}_{3}$ are either 0 or $\mathbf{g}_{i}=h_{i} \Theta_{i}$ with $h_{i}=0$, which implies that $\mathbf{G}_{1}$ can not be expressed as the linear combination of $\mathrm{G}_{2}$ and $\mathrm{G}_{3}$.

Similarly, in order to prove $\mathrm{G}_{2}$ and $\mathrm{G}_{3}$ are independent of the remaining vector groups, we only need to rearrange the channel matrix as

$$
\left\{h_{2}, h_{5}, \cdots, h_{3 p-1}, h_{3 p-2}, h_{3 p-5}, \cdots, h_{1}, h_{3}, h_{6}, \cdots, h_{3 p}\right\}
$$

and

$$
\left\{h_{3}, h_{6}, \cdots, h_{3 p}, h_{3 p-1}, h_{3 p-4}, \cdots, h_{2}, h_{1}, h_{4}, \cdots, h_{3 p-2}\right\}
$$

respectively. Hence, $\mathbf{G}_{1}, \mathrm{G}_{2}$ and $\mathrm{G}_{3}$ are linearly independent vector groups as long as $\mathbf{H} \neq 0$.

According to Proposition 1, the code $\mathrm{C}$ in (35) achieves full diversity using PIC grouping decoding with the group scheme $\mathcal{I}=\left\{\mathcal{I}_{1}, \mathcal{I}_{2}, \mathcal{I}_{3}\right\}$.

Similarly, we can prove that the codes $\mathbf{C}_{3 p-1}$ and $\mathbf{C}_{3 p+1}$ achieve full diversity with PIC grouping decoding. 


\section{Code Design Examples}

For $M=4$. It has $T=7$.

$$
\mathbf{C}_{4}=\left[\begin{array}{cccc}
X_{11} & 0 & 0 & 0 \\
0 & X_{22} & 0 & 0 \\
0 & 0 & X_{33} & 0 \\
X_{21} & 0 & 0 & X_{14} \\
0 & X_{32} & 0 & X_{24} \\
0 & 0 & X_{13} & X_{34} \\
X_{31} & X_{12} & X_{23} & 0
\end{array}\right],
$$

The rate of this code is $12 / 7$. The equivalent channel of the code $\mathbf{C}_{4}$ is

$$
\mathcal{H}=\left[\begin{array}{ccc}
\mathbf{g}_{1} & \mathbf{0} & \mathbf{0} \\
\mathbf{0} & \mathrm{g}_{2} & \mathbf{0} \\
\mathbf{0} & \mathbf{0} & \mathrm{g}_{3} \\
\mathbf{g}_{4} & \mathrm{~g}_{1} & \mathbf{0} \\
\mathbf{0} & \mathrm{g}_{4} & \mathbf{g}_{2} \\
\mathbf{g}_{3} & \mathbf{0} & \mathbf{g}_{4} \\
\mathbf{g}_{2} & \mathbf{g}_{3} & \mathbf{g}_{1}
\end{array}\right]
$$

where the $1 \times 4$ row vector $\mathbf{g}_{i}=\theta_{i} h_{i}, i=1,2,3,4$, with $\theta_{i}$ being the $i$ th row of the matrix $\Theta$. The grouping scheme for the PIC group decoding is $\mathcal{I}_{1}=\{1,2,3,4\}, \mathcal{I}_{2}=\{5,6,7,8\}$ and $\mathcal{I}_{3}=\{9,10,11,12\}$. Obviously, the code can obtain the full diversity with the PIC group decoding. 
For $M=6$. It has $T=10$.

$$
\mathbf{C}_{6}=\left[\begin{array}{cccccc}
X_{1,1} & 0 & 0 & 0 & 0 & 0 \\
0 & X_{2,2} & 0 & 0 & 0 & 0 \\
0 & 0 & X_{3,3} & 0 & 0 & 0 \\
X_{2,1} & 0 & 0 & X_{1,4} & 0 & 0 \\
0 & X_{3,2} & 0 & 0 & X_{2,5} & 0 \\
0 & 0 & X_{1,3} & 0 & 0 & X_{3,6} \\
0 & X_{1,2} & 0 & X_{2,4} & 0 & 0 \\
0 & 0 & X_{2,3} & 0 & X_{3,5} & 0 \\
X_{3,1} & 0 & 0 & 0 & 0 & X_{1,6} \\
0 & 0 & 0 & X_{3,4} & X_{1,5} & X_{2,6}
\end{array}\right]
$$

The rate of this code is $18 / 10$. The equivalent channel of the code $\mathbf{C}_{6}$ is

$$
\mathcal{H}=\left[\begin{array}{ccc}
\mathbf{g}_{1} & \mathbf{0} & \mathbf{0} \\
\mathbf{0} & \mathbf{g}_{2} & \mathbf{0} \\
\mathbf{0} & \mathbf{0} & \mathbf{g}_{3} \\
\mathbf{g}_{4} & \mathbf{g}_{1} & \mathbf{0} \\
\mathbf{0} & \mathbf{g}_{5} & \mathbf{g}_{2} \\
\mathbf{g}_{3} & \mathbf{0} & \mathbf{g}_{6} \\
\mathbf{g}_{2} & \mathbf{g}_{4} & \mathbf{0} \\
\mathbf{0} & \mathbf{g}_{3} & \mathbf{g}_{5} \\
\mathbf{g}_{6} & \mathbf{0} & \mathbf{g}_{1} \\
\mathbf{g}_{5} & \mathbf{g}_{6} & \mathbf{g}_{4}
\end{array}\right]
$$

where the $1 \times 6$ row vector $\mathrm{g}_{i}=\theta_{i} h_{i}, i=1,2,3,4,5,6$, with $\theta_{i}$ being the $i$ th row of the matrix $\Theta$. The grouping scheme for the PIC group decoding is $\mathcal{I}_{1}=\{1,2,3,4,5,6\}, \mathcal{I}_{2}=$ $\{7,8,9,10,11,12\}$ and $\mathcal{I}_{3}=\{13,14,15,16,17,18\}$. Obviously, the code can obtain the full 
diversity with the PIC group decoding.

A code example of $M=9$ is given by

$$
\mathbf{C}_{9}=\left[\begin{array}{ccccccccc}
X_{1,1} & 0 & 0 & 0 & 0 & 0 & 0 & 0 & 0 \\
0 & X_{2,2} & 0 & 0 & 0 & 0 & 0 & 0 & 0 \\
0 & 0 & X_{3,3} & 0 & 0 & 0 & 0 & 0 & 0 \\
X_{2,1} & 0 & 0 & X_{1,4} & 0 & 0 & 0 & 0 & 0 \\
0 & X_{3,2} & 0 & 0 & X_{2,5} & 0 & 0 & 0 & 0 \\
0 & 0 & X_{1,3} & 0 & 0 & X_{3,6} & 0 & 0 & 0 \\
0 & 0 & 0 & X_{2,4} & 0 & 0 & X_{1,7} & 0 & 0 \\
0 & 0 & 0 & 0 & X_{3,5} & 0 & 0 & X_{2,8} & 0 \\
0 & 0 & 0 & 0 & 0 & X_{1,6} & 0 & 0 & X_{3,9} \\
0 & X_{1,2} & 0 & 0 & 0 & 0 & X_{2,7} & 0 & 0 \\
0 & 0 & X_{2,3} & 0 & 0 & 0 & 0 & X_{3,8} & 0 \\
X_{3,1} & 0 & 0 & 0 & 0 & 0 & 0 & 0 & X_{1,9} \\
0 & 0 & 0 & X_{3,4} & X_{1,5} & X_{2,6} & 0 & 0 & 0 \\
0 & 0 & 0 & 0 & 0 & 0 & X_{3,7} & X_{1,8} & X_{2,9}
\end{array}\right] .
$$

\section{Simulation Results}

In this section, simulation results of the proposed STBC with the PIC group decoding over Rayleigh fading channels are presented. We first show bit error rate (BER) performance of the codes proposed in this paper for four transmit antennas and compare them to the one proposed in [38]. Specifically, we consider three STBC for four transmit antennas proposed in this paper, i.e., $\mathbf{C}_{4,6,3}$ in (31), $\mathbf{C}_{4,6,2}$ in (29) and $\mathbf{C}_{4,5,2}$ in (26), and then compare them with Guo-Xia's code given in [38, Section VI - Example 2]. In order to make a fair performance comparison, we keep the same bandwidth efficiency of $8 \mathrm{bps} / \mathrm{Hz}$. Thus, we use 16QAM for the code $\mathrm{C}_{4,6,3}$ (the code rate is $R=2$ ) and 64QAM for both codes $\mathbf{C}_{4,6,2}$ (the code rate is $R=\frac{4}{3}$ ) and Guo-Xia's code (the code rate is $R=\frac{4}{3}$ ). For the code $\mathrm{C}_{4,5,2}$, because it has a code rate $R=\frac{8}{5}$ we use 64QAM and thus its bandwidth efficiency is $9.6 \mathrm{bps} / \mathrm{Hz}$ higher than the other three codes. Since we use square QAM, in the rotation matrix $\Theta$ we use $m=4$ in Example 1 of Section III.

Fig. 1 shows the performance of the proposed code $\mathrm{C}_{4,5,2}$ with various detection approaches for a $4 \times 4$ MIMO system over Rayleigh fading channels. It can be seen that the code with 
PIC and PIC-SIC group decoding algorithms has both less than $1 \mathrm{~dB}$ SNR performance loss compared to the one with the ML decoding. The performance loss of the PIC and PIC-SIC group decoding is trade for the largely reduced decoding complexity. Specifically, for 16QAM signaling the ML decoding complexity is $\mathcal{O}_{M L}=16^{8}=2^{32}$ and the complexity of the PIC group decoding is $\mathcal{O}_{P I C}=2 \times 16^{4}=2^{17}$. Moreover, it is obvious that the code $\mathrm{C}_{4,5,2}$ with the PIC group decoding and the PIC-SIC group decoding can both obtain full diversity at high SNR. BLAST and ZF detection of the code $\mathrm{C}_{4,5,2}$ cannot obtain full diversity.

Fig. 2 shows the performance of the proposed code $\mathbf{C}_{4,6,3}$ with various detection approaches for a $4 \times 4$ MIMO system over Rayleigh fading channels. It is shown that the code $\mathbf{C}_{4,6,3}$ with PIC group decoding does not guarantee the full diversity at high SNR. However, with PIC-SIC group decoding the code $\mathbf{C}_{4,6,3}$ can achieve the full diversity. The performance gap between the ML decoding and the PIC group decoding is around $2 \mathrm{~dB}$ SNR loss, but the decoding complexity is significantly reduced by the PIC group decoding from $16^{12}$ to $3 \times 16^{4}$. Again, ZF and BLAST detection cannot obtain the full diversity.

Fig. 3 presents the performance comparison between TAST code [20] and the proposed code $\mathrm{C}_{4,6,3}$ with ML, PIC and PIC-SIC group decoding algorithms, respectively, for $4 \times 4$ systems over Rayleigh fading channels. Note the rate of TAST code is full, i.e., 4 symbols per channel use for 4 transmit antennas. To keep the bandwidth efficiency of $8 \mathrm{bps} / \mathrm{Hz}, 4-\mathrm{QAM}$ is used for TAST code and 16-QAM is used for the code $\mathbf{C}_{4,6,3}$. It is shown in Fig. 3 that TAST code with the ML decoding gives the best performance. However, with PIC and PIC-SIC group decoding algorithms TAST code will lose full diversity. The proposed code $\mathbf{C}_{4,6,3}$ can obtain much better performance than TAST code when both use the PIC and PIC-SIC group decoding algorithms.

Fig. 4 gives the performance comparison between Perfect STBC [24] and the proposed code $\mathrm{C}_{4,6,3}$ with ML, PIC and PIC-SIC group decoding algorithms, respectively, for $4 \times 4$ systems over Rayleigh fading channels. Similar to TAST code, Perfect STBC cannot obtain full diversity with the PIC and PIC-SIC group decoding. The proposed code $\mathbf{C}_{4,6,3}$ has a much better performance than Perfect STBC while admitting low complexity in the decoding.

Fig. 5 shows the BER performance of various codes with the PIC group decoding for 4 transmit and 4 receive antennas. It can be seen that the code $\mathbf{C}_{4,6,2}$ has very similar performance to Guo-Xia's code. Moreover, the code $\mathrm{C}_{4,5,2}$ has $1 \mathrm{~dB}$ loss compared to Guo-Xia's code. This is because it has a higher bandwidth efficiency than Guo-Xia's code. In particular, the code $\mathbf{C}_{4,6,3}$ 
in (31) achieves the best BER performance among all the simulated codes. This is attributed to more information symbols embedded in the code $\mathbf{C}_{4,6,3}$ than the other codes and its code rate is the highest.

We also consider the simulation of the proposed code $\mathrm{C}_{5,6,2}$ in (33) with the PIC group decoding for five transmit antennas. Fig. 6 shows the BER performance of the code $\mathbf{C}_{5,6,2}$ for three, four and five receive antennas, respectively. It demonstrates that an increase of the number of receive antennas results in a larger diversity gain as illustrated by the slope of the BER curves.

\section{CONCLUSION}

In this paper, two designs of STBC that can achieve full diversity with PIC group decoding was proposed. The first proposed STBC are constructed with multiple diagonal layers and each layer is composed of a fixed number of coded symbols equal to $M$, i.e., the number of the transmit antennas. The code rate of the first proposed STBC is varied in accordance with the number of layers embedded in the codeword. For the PIC group decoding, a grouping scheme was proposed to cluster every $M$ neighboring columns of the equivalent channel matrix into one group. With the proposed STBC and the PIC group decoding in MIMO systems, it was proved that full diversity can be achieved when two diagonal layers are embedded in the code matrix. Moreover, for the full-diversity STBC with the PIC group decoding, the code rate is up to 2 symbols per channel use. When PIC-SIC group decoding is used, the proposed full-diversity STBC can have a rate up to $M$. A few examples of code design achieving full diversity PIC group decoding were given. The second proposed STBC are based on three-layers and can achieve full diversity with the PIC group decoding and their code rates can be up to $9 / 4$. Simulation results confirmed the theoretical analysis and show the full diversity performance of the proposed codes when the PIC group decoding is applied at receiver. It was also demonstrated that the proposed STBC outperform Perfect STBC and TAST code when PIC group decoding is applied. 


\section{APPENDIX I - PROOF OF LEMMA 1}

\section{A. Proof of Lemma 1.1}

Proof: Define the $T \times M$ matrix $\mathbf{C}_{p}(p=1,2, \cdots, P)$ as follows,

$$
\mathbf{C}_{p}=\left[\begin{array}{c}
\mathbf{0}_{(p-1) \times M} \\
\operatorname{diag}\left(\mathbf{X}_{p}\right) \\
\mathbf{0}_{(P-p) \times M}
\end{array}\right], p=1,2, \cdots, P,
$$

where $P=T-M+1$ and $\mathbf{X}_{p}=\left[\begin{array}{llll}X_{p, 1} & X_{p, 2} & \cdots & X_{p, M}\end{array}\right]^{t}$ is given by (17). Then, (16) can be written as

$$
\mathbf{C}=\sum_{p=1}^{P} \mathbf{C}_{p}
$$

For MISO systems, we have $\mathbf{H}=\mathbf{h}=\left[\begin{array}{llll}h_{1} & h_{2} & \cdots & h_{M}\end{array}\right]^{t}$ with $h_{m}(m=1,2 \cdots, M)$ being the channel gain from the $m$-th transmit antenna to the receiver. Using (44), we can express (1) as

$$
\begin{aligned}
\mathbf{y} & =\sqrt{\frac{\rho}{\mu}} \sum_{p=1}^{P} \mathbf{C}_{p} \mathbf{h}+\mathbf{w} \\
& =\sqrt{\frac{\rho}{\mu}} \sum_{p=1}^{P} \mathcal{H}_{p} \mathbf{X}_{p}+\mathbf{w}
\end{aligned}
$$

where $\mathbf{y} \in \mathbb{C}^{T \times 1}, \mathbf{w} \in \mathbb{C}^{T \times 1}$ and $\mathcal{H}_{p} \in \mathbb{C}^{T \times M}$ is given by

$$
\mathcal{H}_{p}=\left[\begin{array}{c}
\mathbf{0}_{(p-1) \times M} \\
\operatorname{diag}(\mathbf{h}) \\
\mathbf{0}_{(P-p) \times M}
\end{array}\right], p=1,2, \cdots, P .
$$

Using (17), we can further write (45) as

$$
\begin{aligned}
\mathbf{y} & =\sqrt{\frac{\rho}{\mu}} \sum_{p=1}^{P} \mathcal{H}_{p} \boldsymbol{\Theta} \mathbf{s}_{p}+\mathbf{w} \\
& =\sqrt{\frac{\rho}{\mu}} \mathcal{H} \mathbf{s}+\mathbf{w},
\end{aligned}
$$

where the equivalent channel matrix $\mathcal{H} \in \mathbb{C}^{T \times M P}$ is given by

$$
\mathcal{H}=\left[\begin{array}{llll}
\left(\mathcal{H}_{1} \Theta\right) & \left(\mathcal{H}_{2} \Theta\right) & \cdots & \left(\mathcal{H}_{P} \Theta\right)
\end{array}\right]
$$


and $\mathbf{s}=\left[\begin{array}{llll}\mathbf{s}_{1}^{t} & \mathbf{s}_{2}^{t} & \cdots & \mathbf{s}_{P}^{t}\end{array}\right]^{t}$.

Let $\mathbf{G}_{p}=\mathcal{H}_{p} \boldsymbol{\Theta}$ for $p=1,2, \cdots, P$. Using (46), we get

$$
\mathbf{G}_{p}=\left[\begin{array}{c}
\mathbf{0}_{(p-1) \times M} \\
\operatorname{diag}(\mathbf{h}) \Theta \\
\mathbf{0}_{(P-p) \times M}
\end{array}\right], \quad p=1,2, \cdots, P .
$$

Then, (48) can be written as

$$
\mathcal{H}=\left[\begin{array}{llll}
\mathbf{G}_{1} & \mathbf{G}_{2} & \cdots & \mathbf{G}_{P}
\end{array}\right] .
$$

\section{B. Proof of Lemma 1.2}

Proof: Next, we shall prove that $\mathbf{G}_{1}$ and $\mathbf{G}_{2}$ are linearly independent vector groups as long as $\mathbf{h} \neq \mathbf{0}$. To do so, we may need the following definitions.

Definition 3: [38]. Let $\mathcal{V}=\left\{\mathbf{v}_{i} \in \mathbb{C}^{n}, i=0,1, \cdots, k-1\right\}$ be a set of vectors. Vector $\mathbf{v}_{k}$ is said to be independent of $\mathcal{V}$ if for any $a_{i} \in \mathbb{C}, i=0,1, \cdots, k-1$,

$$
\mathbf{v}_{k}-\sum_{i=0}^{k-1} a_{i} \mathbf{v}_{i} \neq \mathbf{0} .
$$

Definition 4: [38]. Let $\mathcal{V}_{0}, \mathcal{V}_{1}, \cdots, \mathcal{V}_{n-1}, \mathcal{V}_{n}$ be $n+1$ groups of vectors. Vector group $\mathcal{V}_{n}$ is said to be independent of $\mathcal{V}_{0}, \mathcal{V}_{1}, \cdots, \mathcal{V}_{n-1}$ if every vector in $\mathcal{V}_{n}$ is independent of $\bigcup_{i=0}^{n-1} \mathcal{V}_{i}$.

Definition 5: [38]. Let $\mathcal{V}_{0}, \mathcal{V}_{1}, \cdots, \mathcal{V}_{n-1}, \mathcal{V}_{n}$ be $n+1$ groups of vectors. The vector groups $\mathcal{V}_{0}, \mathcal{V}_{1}, \cdots, \mathcal{V}_{n-1}, \mathcal{V}_{n}$ are said to be linearly independent if for $0 \leq k \leq n, \mathcal{V}_{k}$ is independent of the remaining vector groups $\mathcal{V}_{0}, \mathcal{V}_{1}, \cdots, \mathcal{V}_{k-1}, \mathcal{V}_{k+1}, \cdots, \mathcal{V}_{n}$

To prove that $\mathbf{G}_{1}$ and $\mathbf{G}_{2}$ are linearly independent, from Definition $[$ it is equivalent to prove the following two steps:

1) Prove that $G_{1}$ is independent of $G_{2}$ for any $\mathbf{h} \neq \mathbf{0}$.

2) Prove that $G_{2}$ is independent of $G_{1}$ for any $\mathbf{h} \neq \mathbf{0}$.

Using (23), we can express $G_{1}$ and $G_{2}$ as, respectively,

$$
\mathbf{G}_{1}=\left[\begin{array}{c}
\operatorname{diag}(\mathbf{h}) \boldsymbol{\Theta} \\
\mathbf{0}_{1 \times M}
\end{array}\right], \quad \mathbf{G}_{2}=\left[\begin{array}{c}
\mathbf{0}_{1 \times M} \\
\operatorname{diag}(\mathbf{h}) \boldsymbol{\Theta}
\end{array}\right] .
$$


Step $1-\mathbf{G}_{1}$ is independent of $\mathbf{G}_{2}$ for any $\mathbf{h} \neq \mathbf{0}$.

For any $\mathbf{h} \neq \mathbf{0}$, we can find a minimal index $\sigma(1 \leq \sigma \leq M)$ such that $h_{\sigma} \neq 0$. That is, $h_{1}=\cdots=h_{\sigma-1}=0$.

Let $\mathbf{g}_{1, m}$ be the $m$-th $(1 \leq m \leq M)$ column of the matrix $\mathbf{G}_{1}$. In order to prove that $\mathbf{G}_{1}$ is independent of $\mathbf{G}_{2}$, from Definition 4 we see it is equivalent to prove that the vector $\mathbf{g}_{1, m}$ is independent of $\mathbf{G}_{2}$ for all $m=1,2, \cdots, M$. Further, using Definition 3 it is equivalent to prove that

$$
\mathbf{g}_{1, m}-\mathbf{G}_{2} \bar{\beta}_{2} \neq \mathbf{0}, \quad \forall m, m=1,2, \cdots, M,
$$

where $\bar{\beta}_{2}$ denotes an $M \times 1$ vector. Equivalently, (52) can be expressed as

$$
a \mathbf{g}_{1, m}-\mathbf{G}_{2} a \bar{\beta}_{2} \neq \mathbf{0}, \quad \forall m, m=1,2, \cdots, M,
$$

where $a$ is a constant and $a \neq 0$.

In order to prove (53), we can use proof by contradiction. That is, we assume that $a \mathbf{g}_{1, m}-$ $\mathbf{G}_{2} a \bar{\beta}_{2}=\mathbf{0}, \forall m, m=1,2, \cdots, M$, and $a \neq 0$. Then, we examine the $\sigma$-th equation (from top to bottom) of (53) and get $a g_{\sigma, m}^{(1)}=0$ for $1 \leq m \leq M$, where $g_{\sigma, m}^{(1)}$ denotes the $(\sigma, m)$-th entry of the matrix $\mathbf{G}_{1}$. This is because all top $\sigma$ rows of $\mathbf{G}_{2}$ are all zeros when $h_{1}=\cdots=h_{\sigma-1}=0$ as seen from (23). Again from (23), we have $g_{\sigma, m}^{(1)}=h_{\sigma} \theta_{\sigma, m}$ where $\theta_{\sigma, m}$ denotes the $(\sigma, m)$-th entry of the matrix $\Theta$. For given $h_{\sigma} \neq 0$ and $\theta_{\sigma, m} \neq 0$ for all $m=1,2, \cdots, M$, we get $a=0$. This contradicts with the assumption $a \neq 0$. Therefore, (53) holds and Step 1 is proved.

Step $2-\mathbf{G}_{2}$ is independent of $\mathbf{G}_{1}$ for any $\mathbf{h} \neq \mathbf{0}$.

For any $\mathbf{h} \neq \mathbf{0}$, we can have a maximal index $v(1 \leq v \leq M)$ such that $h_{v} \neq 0$. That is, $h_{v+1}=\cdots=h_{M}=0$.

Following the similar way in the proof of Step 1, we can prove Step 2 as follows.

Let $\mathbf{g}_{2, m}$ denote the $m$-th $(1 \leq m \leq M)$ column of $\mathbf{G}_{2}$. In order to prove that $\mathbf{G}_{2}$ is independent of $\mathbf{G}_{1}$, from Definition 4 it is equivalent to prove that the vector $\mathbf{g}_{2, m}$ is independent of $\mathbf{G}_{1}$ for all $m=1,2, \cdots, M$. Further, using Definition 3 it is equivalent to prove that

$$
\mathbf{g}_{2, m}-\mathbf{G}_{1} \bar{\beta}_{1} \neq \mathbf{0}, \quad \forall m, m=1,2, \cdots, M,
$$


where $\bar{\beta}_{1}$ denotes an $M \times 1$ vector. Equivalently, (54) can be expressed as

$$
a \mathbf{g}_{2, m}-\mathbf{G}_{1} a \bar{\beta}_{1} \neq \mathbf{0}, \quad \forall m, m=1,2, \cdots, M,
$$

where $a$ is a constant and $a \neq 0$.

In order to prove (55), we can use proof by contradiction. We assume that $a \mathbf{g}_{2, m}-\mathbf{G}_{1} a \bar{\beta}_{1}=$ $\mathbf{0}, \forall m, m=1,2, \cdots, M$, and $a \neq 0$. Note the first row of $\mathbf{G}_{2}$ is all-zero. Then, we examine the $(v+1)$-th equation(from top to bottom) of (55) and get $a g_{v+1, m}^{(2)}=0$ for $1 \leq m \leq M$, where $g_{v+1, m}^{(2)}$ denotes the $(v+1, m)$-th entry of the matrix $\mathbf{G}_{2}$. This is because the $(v+1)$-th row of $\mathbf{G}_{1}$ is all-zero when $h_{v+1}=\cdots=h_{M}=0$ as seen from (23). Again from (23), we have $g_{v+1, m}^{(2)}=h_{v} \theta_{v, m}$ where $\theta_{v, m}$ denotes the $(v, m)$-th entry of the matrix $\Theta$. For given $h_{v} \neq 0$ and $\theta_{v, m} \neq 0$ for all $m=1,2, \cdots, M$, we then get $a=0$. This contradicts with the assumption $a \neq 0$. Therefore, (55) holds and Step 2 is proved.

To summarize Step 1 and Step 2, we prove that $\mathbf{G}_{1}$ and $\mathbf{G}_{2}$ are linearly independent vector groups for any $\mathbf{h} \neq \mathbf{0}$.

\section{REFERENCES}

[1] S. M. Alamouti, "A simple transmit diversity technique for wireless communication," IEEE J. Sel. Areas Commun., vol. 16, pp. 1451-1458, Oct. 1998.

[2] V. Tarokh, H. Jafarkhani, and A. R. Calderbank, "Space-time block codes from orthogonal designs," IEEE Trans. Inf. Theory, vol. 45, pp. 1456-1467, July 1999.

[3] X. Liang, "Orthogonal designs with maximal rates," IEEE Trans. Inf. Theory, vol. 49, pp. 2468-2503, Oct. 2003.

[4] W. Su, X.-G. Xia, and K. J. R. Liu, "A systematic design of high-rate complex orthogonal space-time block codes," IEEE Commun. Lett., vol. 8, no. 6, pp.380-382, June 2004.

[5] K. Lu, S. Fu, and X.-G. Xia, "Closed form designs of complex orthogonal space-time block codes of rates $(k+1) /(2 k)$ for $2 k-1$ or $2 k$ transmit antennas," IEEE Trans. Inf. Theory, vol. 51, pp. 4340-4347, Dec. 2005.

[6] H. Wang and X.-G Xia, "Upper bounds of rates of complex orthogonal space-time block codes," IEEE Trans. Inf. Theory, vol 49, pp. 2788-2796, Oct. 2003.

[7] H. Jafarkhani, “A quasi-orthogonal space-time block code," IEEE Trans. Commun., vol. 49, pp. 1-4, Jan. 2001.

[8] O. Tirkkonen, A. Boariu, and A. Hottinen, "Minimal non-orthogonality rate 1 space-time block code for 3+ Tx antennas," in Proc. IEEE 6th Int. Symp. on Spread-Spectrum Tech. and Appl. (ISSSTA 2000), Sep. 2000, pp. 429-432.

[9] C. B. Papadias and G. J. Foschini, "Capacity-approaching space-time codes for systems employing four transmit antennas," IEEE Trans. Inf. Theory, vol. 49, pp. 726-733, Mar. 2003.

[10] O. Tirkkonen, "Optimizing space-time block codes by constellation rotations," Proc. Finnish Wireless Commun. Workshop, Finland, pp. 59-60, Oct. 2001.

[11] N. Sharma and C. B. Papadias, "Full-rate full-diversity linear quasi-orthogonal space-time codes for any number of transmit antennas," EURASIP J. Applied Signal Processing, vol. 9, pp. 1246-1256, Aug. 2004. 
[12] W. Su and X.-G. Xia, "Signal constellations for quasi-orthogonal space-time block codes with full diversity," IEEE Trans. Inform. Theory, vol. 50, pp. 2331-2347, Oct. 2004.

[13] M. Janani and A. Nosratinia, "Efficient space-time block codes derived from quasi-orthogonal structures," IEEE Trans. Wireless Commun., vol. 6, pp. 1643-1646, May 2007.

[14] Z. A. Khan and B. S. Rajan, "Single-symbol maximum-likelihood decodable linear STBCs," IEEE Trans. Inf. Theory, vol. 52, pp. 2062-2091, May 2006.

[15] C. Yuen, Y. Guan, and T. T. Tjhung, "Quasi-orthogonal STBC with minimum decoding complexity," IEEE Trans. Wireless Commun., vol. 4, pp. 2089-2094, Sep. 2005.

[16] H. Wang, D. Wang, and X.-G. Xia, "On optimal quasi-orthogonal space-time block codes with minimum decoding complexity," IEEE Trans. Inf. Theory, vol. 55, pp. 1104-1130, Mar. 2009.

[17] D. N. Dao, C. Yuen, C. Tellambura, Y. L. Guan, and T. T. Tjhung, "Four-group decodable space-time block codes," IEEE Trans. Signal Processing, vol. 56, pp. 424-430, Jan. 2008.

[18] S. Karmakar and B. S. Rajan, "Multigroup decodable STBCs from clifford algebras," IEEE Trans. Inf. Theory, vol. 55, pp. 223-231, Jan. 2009.

[19] M. O. Damen, A. Tewfik, and J. C. Belfiore, "A construction of a space-time code based on number theory," IEEE Trans. Inf. Theory, vol. 48, pp. 753-760, Mar. 2002.

[20] H. El Gamal and M. O. Damen, “Universal space-time coding,” IEEE Trans. Inf. Theory, vol. 49, pp. 1097-1119, May 2003.

[21] B. A. Sethuraman, B. S. Rajan, and V. Shashidhar, "Full-diversity, high-rate space-time block codes from division algebras," IEEE Trans. Inf. Theory, vol. 49, pp. 2596-2616, Oct. 2003.

[22] Kiran T. and B. S. Rajan, "STBC-scheme with nonvanishing determinant for certain number of transmit antennas," IEEE Trans. Inf. Theory, vol. 51, pp. 2984-2992, Aug. 2005.

[23] P. Elia, K. R. Kumar, S. A. Pawar, P. V. Kumar, and H.-F. Lu, "Explicit space-time codes achieving the diversity-multiplexing gain tradeoff," IEEE Trans. Inf. Theory, vol. 52, pp. 3869-3884, Sep. 2006.

[24] F. Oggier, G. Rekaya, J.-C. Belfiore, and E. Viterbo, "Perfect space-time block codes," IEEE Trans. Inf. Theory, vol. 52, pp. 3885-3902, Sep. 2006.

[25] P. Elia, B. A. Sethuraman, and P. V. Kumar, "Perfect space-time codes for any number of antennas," IEEE Trans. Inf. Theory, vol. 53, pp. 3853-3868,Nov. 2007.

[26] F. Oggier and E. Viterbo, "Algebraic number theory and code design for Rayleigh fading channels," Foundations and Trends in Communications and Information Theory, vol. 1, no. 3, pp. 333-415.

[27] F. Oggier, J.-C. Belfiore, and E. Viterbo, "Cyclic division algebras: A tool for space-time coding," Foundations and Trends in Communications and Information Theory, vol. 4, no. 1, pp. 1-95.

[28] E. Viterbo and J. Boutros, "A universal lattice code decoder for fading channels," IEEE Trans. Inf. Theory, vol. 45, pp. 1639-1642, July 1999.

[29] J. Jalden and B. Ottersten, "On the complexity of sphere decoding in digital communications," IEEE Trans. Signal Process., vol. 53, pp. 1474-1484, Apr. 2005.

[30] X. H. Nguyen and J. Choi, "Joint design of groupwise STBC and SIC based receiver," IEEE Commun. Lett., vol. 12, pp. 115-117, Feb. 2008.

[31] E. Biglieri, Y. Hong, and E. Viterbo, "On fast-decodable space-time block codes," IEEE Trans. Inf. Theory, vol. 55, pp. 524-530, Feb. 2009. 
[32] A. Hedayat and A. Nosratinia, "Outage and diversity of linear receivers in flat-fading MIMO channels," IEEE Trans. Signal Processing, vol. 55, pp. 5868-5873, Dec. 2007.

[33] J. Liu, J.-K. Zhang, and K. M. Wong, "Full-diversity codes for MISO systems equipped with linear or ML detectors," IEEE Trans. Inf. Theory, vol. 54, pp. 4511-4527, Oct. 2008.

[34] Y. Shang and X.-G. Xia, "Space-time block codes achieving full diversity with linear receivers," IEEE Trans. Inf. Theory, vol. 54, pp. 4528-4547, Oct. 2008.

[35] W. Zhang and J. Yuan, "A simple design of space-time block codes achieving full diversity with linear receivers," in Proc. IEEE ICASSP, Taipei, Apr. 20-24, 2009, pp. 2729-2732.

[36] W. Zhang and J. Yuan, "Linear receiver based high-rate space-time block codes," in Proc. IEEE ISIT, Seoul, Korea, June 29-July 3, 2009, pp. 94-98.

[37] H. Wang, X.-G. Xia, Q. Yin, and B. Li, "A family of space-time block codes achieving full diversity with linear receivers," IEEE Trans. Commun., vol. 57, pp. 3607-3617, Dec. 2009.

[38] X. Guo and X.-G. Xia, "On full diversity space-time block codes with partial interference cancellation group decoding," IEEE Trans. Inf. Theory, vol. 55, pp. 4366-4385, Oct. 2009.

[39] M. O. Damen and N. C. Beaulieu, "On diagonal algebraic space-time block codes," IEEE Trans. Commun., vol. 51, pp. 911-919, June 2003.

[40] G. Wang, H. Liao, H. Wang, and X.-G. Xia, "Systematic and optimal cyclotomic lattices and diagonal space-time block code designs," IEEE Trans. Inf. Theory, vol. 50, pp. 3348-3360, Dec. 2004.

[41] G. J. Foschini, "Layered space-time architecture for wireless communication in a fading environment when using multielement antennas," Bell Labs Tech. J., vol. 1, no. 2, pp. 41-59, 1996. 


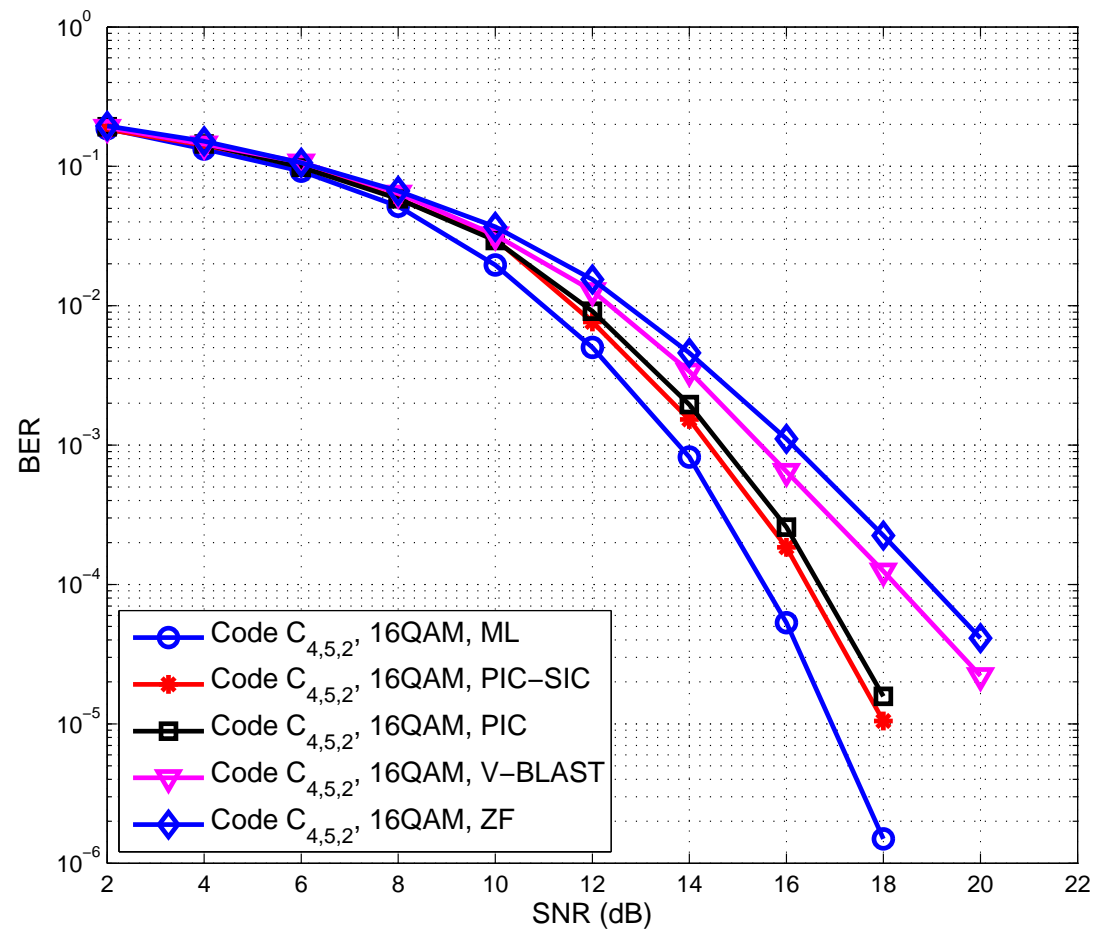

Fig. 1. BER performance of the proposed code $\mathbf{C}_{4,5,2}$ with various detection methods over $4 \times 4$ Rayleigh fading channels. 


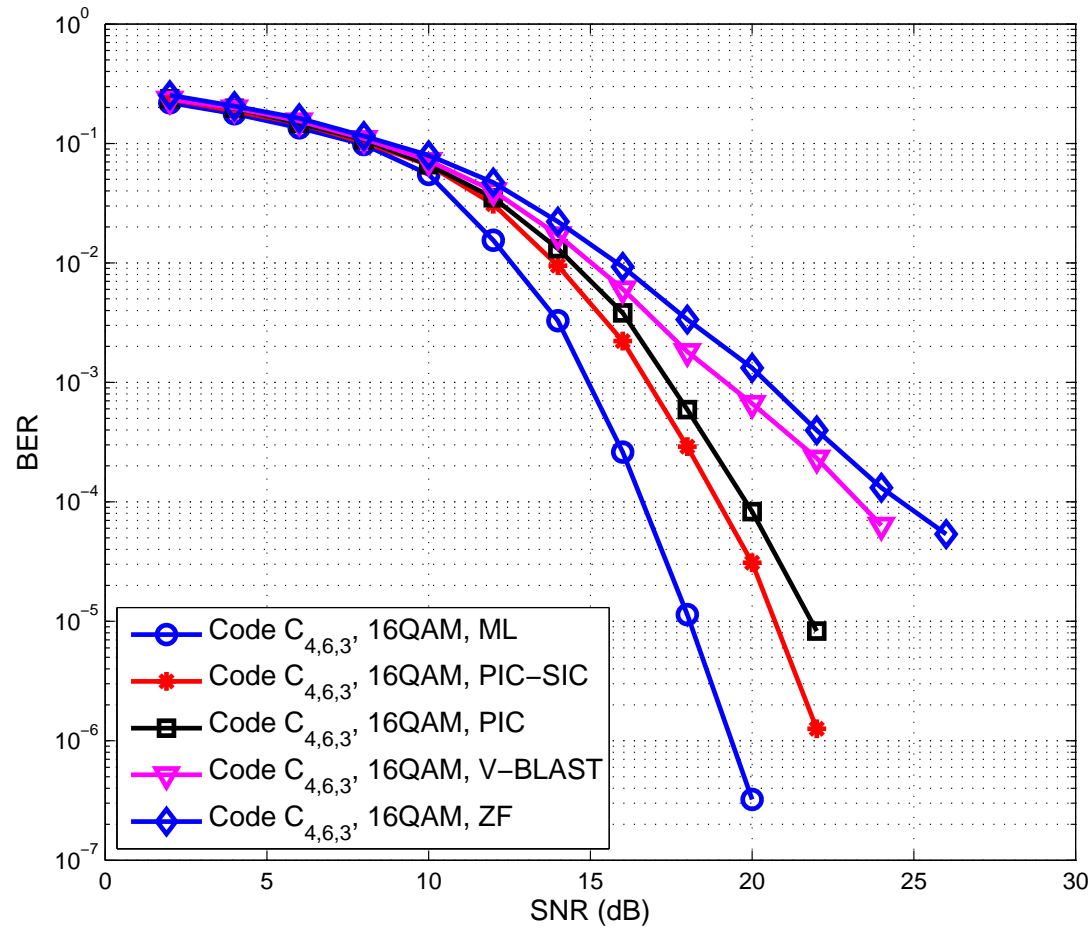

Fig. 2. BER performance of the proposed code $\mathbf{C}_{4,6,3}$ with various detection methods over $4 \times 4$ Rayleigh fading channels. 


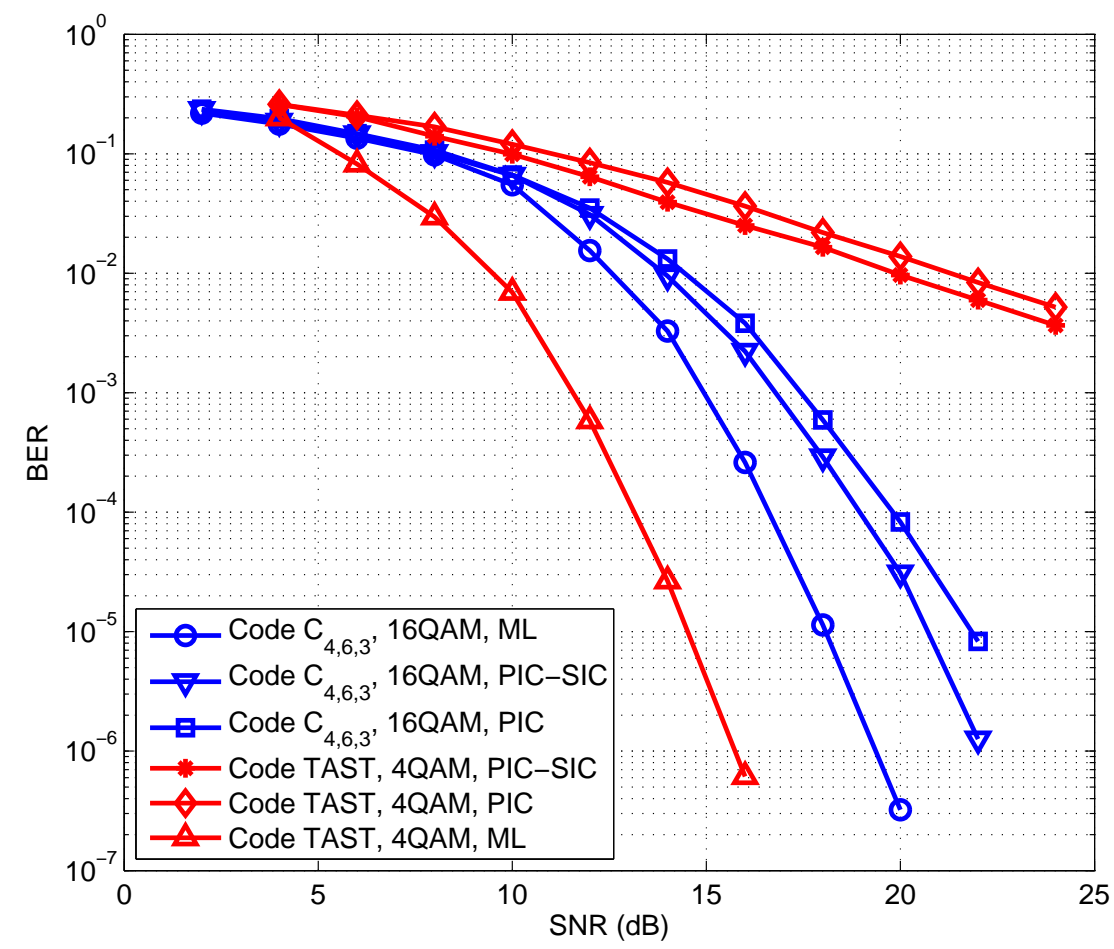

Fig. 3. Performance comparison between the proposed code $\mathbf{C}_{4,6,3}$ and the TAST code [20] for a MIMO system with 4 transmit antennas and 4 receive antennas at $8 \mathrm{bps} / \mathrm{Hz}$. 


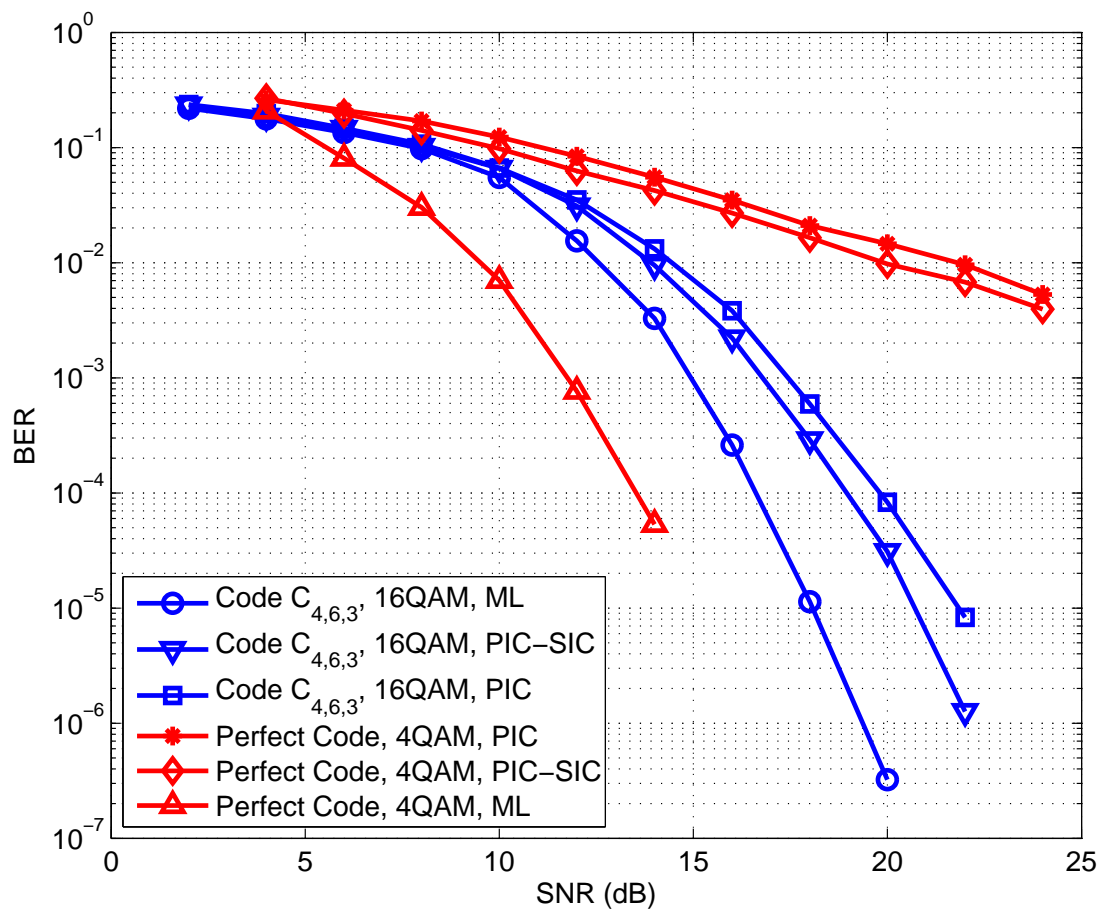

Fig. 4. Performance comparison between the proposed code $\mathbf{C}_{4,6,3}$ and the perfect ST code [24] for a MIMO system with 4 transmit antennas and 4 receive antennas at $8 \mathrm{bps} / \mathrm{Hz}$. 


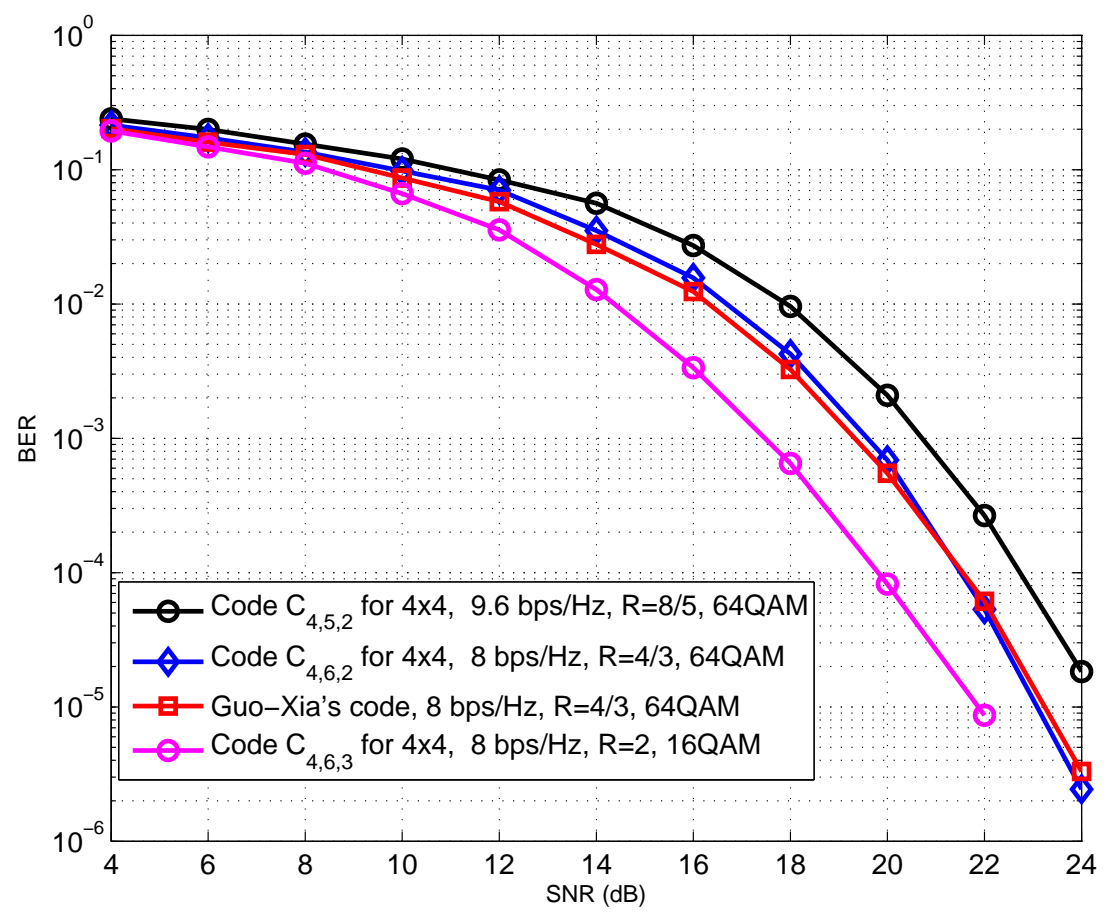

Fig. 5. BER performance of various codes with PIC group decoding for a MIMO system with 4 transmit antennas and 4 receive antennas. 


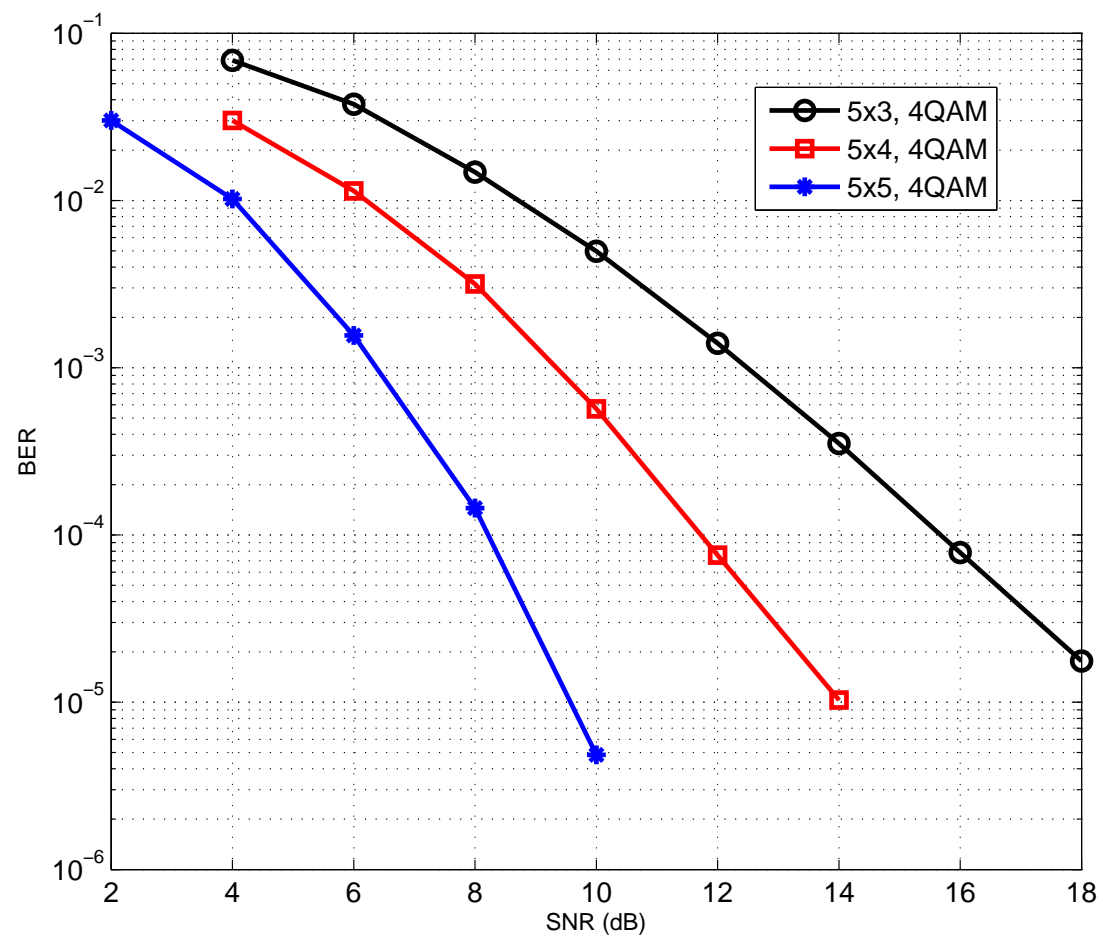

Fig. 6. BER performance of the proposed code $\mathbf{C}_{5,6,2}$ with PIC group decoding for a MIMO system with 5 transmit antennas and different number of receive antennas. 